\title{
REPRESENTATIONS OF TWISTED GROUP ALGEBRAS
}

\author{
BY \\ ROBERT C. BUSBY AND HARVEY A. SMITH
}

\begin{abstract}
We construct a general class of Banach algebras which include as special cases the group algebra of a locally compact group, the group algebra of a group extension (in terms of the subgroup and quotient group), and some other examples, special cases of which have been studied under the name of covariance algebras. We develop the general representation theory and generalize Mackey's theory of induced representations.
\end{abstract}

1. Introduction. In this paper we begin the investigation of a very general class of algebras of functions on locally compact groups taking values in a Banach algebra. A number of authors have investigated special cases of such algebras, notably G. P. Johnson [12], J. Glimm [8], E. Effros and F. Hahn [6], Turumaru [23], Zeller-Meier [26], I. Segal [20], and Doplicher, Kastler and Robinson [5]. Of these, Zeller-Meier's algebras come closest in generality to those we discuss. $\mathrm{He}$ limits himself to discrete groups and $C^{*}$-algebras, and in this context he formulates many of the important concepts. While a number of our results generalize his, our techniques are almost always different. Horst Leptin in [14] (see also [13] and [15]) defines an essentially equivalent class of algebras, however he confines himself to functions taking values in commutative algebras. Our construction differs from his in a number of technical details (we have tried to use concepts and notations more in agreement with previous work on special cases, and usual conventions), and there is very little overlap in subject matter. His representation theory and ours are distinct.

Several authors have studied what are essentially representations of the algebras we discuss, but without defining the algebras. This is true of G. Mackey [16], [17], [18], M. Takesaki [22], Doplicher, Kastler and Robinson [5], and a number of other authors.

We restrict our interest in the present paper to the representation theory of the algebras, leaving functorial considerations and their connection with the ideas centered around induced representations, as well as the detailed study of special cases and the structure theory to a later occasion.

In $\$ 2$, we introduce a few needed concepts and cite some results for later use.

Received by the editors November 29, 1968 and, in revised form, November 13, 1969.

AMS Subject Classifications. Primary 4680; Secondary 4660, 2260.

Key Words and Phrases. Twisted group algebra, covariance algebra, covariance representation, induced representation, group extension.

Copyright (C) 1970, American Mathematical Society 
We then define a twisted group algebra and cite some examples, in particular the group algebra of a group extension. To avoid interrupting the exposition with lengthy computations, the tedious and rather complicated demonstrations of the statements made in $\$ 2$ are delayed to $\S 6$. In $\S 3$ we discuss representations of the twisted group algebras and their relationship to representations of the group and of the range algebra. Some consequences of the results and some special cases are discussed. Representations induced by a representation of the range algebra are discussed in $\$ 4$ and certain canonical representations in $\$ 5$. The more tedious computations are grouped together in $\$ 6$.

We make the convention now that throughout this paper, all algebras will be separable and all topological groups will be second-countable and Hausdorff. We will omit these adjectives throughout the rest of the paper. It is quite likely that most of our results extend in some form to nonseparable cases, but the pathological nature of measures on nonsecond-countable locally compact spaces would necessitate a painful reexamination of concepts and theorems which are well known in the second countable case, and we feel that such a procedure would unnecessarily lengthen and insufficiently improve the results. Those interested can see for themselves which results might perhaps be extended. The difficulties frequently center about such things as the proper choice of a $\sigma$-ring to call the Borel sets.

2. Twisted group algebras. We will need the theory of the Bochner integral as developed in [10]. By using the form of the definition in which a function from a measure space to a Banach algebra is Bochner-integrable when it is the limit a.e. of a sequence of "step functions" (taking countably many values and constant on measurable sets) and the integral of its norm exists, one can easily deduce:

Remark 1. If $f: X \rightarrow A$ and $g: X \rightarrow B$ are Bochner-integrable, $C$ is a Banach space and $\varphi: A \times B \rightarrow C$ is a continuous bilinear functional then $\varphi(f(x), g(x))$ is Bochner-integrable.

It is well known (see [10]) that:

REMARK 2. The set of Bochner-integrable functions from a measure space to a Banach space form a Banach space, the norm of a function being the integral of the norm of its value. This space is the completion with respect to the greatest cross-norm of the tensor product of the range Banach space with the space of integrable complex-valued functions on the measure space (see [9], [21]).

By approximate identity of a Banach*-algebra, we will always mean a two-sided, selfadjoint approximate identity of norm 1 . If $A$ is a Banach*-algebra with an approximate identity and $M(A)$ is its double centralizer algebra [11], we need:

REMARK 3. $A$ is canonically embedded as a closed two-sided ideal in $M(A)$ by means of left and right multiplication. Any automorphism $T$ of $A$ lifts canonically to $M(A)$ and is defined by

$$
T(m) T(a)=T(m a) \text { for } m \in M(A), a \in A .
$$

Let $G$ be a locally compact group with left Haar measure $d g$ and modular 
function $\Delta$. Let $A$ be a Banach*-algebra with $\|x\|=\left\|x^{*}\right\|$ for all $x \in A$ and with an approximate identity.

Let $\mathscr{U}$ be the group of unitaries in $M(A)$, that is the elements $x$ such that $1=x^{*} x$ $=x x^{*}$, and $\|x\|=\left\|x^{*}\right\|=1$, and let $\operatorname{Aut}_{1}(A)$ be the subgroup of the group Aut $(A)$ of continuous *-automorphisms of $A$ such that $T \in \operatorname{Aut}_{1}(A)$ if and only if $\|T\|$ $=\left\|T^{-1}\right\|=1$. A function $f: G \rightarrow A$ is said to be strongly measurable if it is the limit a.e. of: "step functions" as in the definition of the Bochner integral. We will always want Borel measurability and we will use the word Borel for Borel measurable.

Definition 2.1. A twisting pair $(T, \alpha)$ for $G$ and $A$ is a pair of mappings $T: G \rightarrow \operatorname{Aut}_{1}(A), \alpha: G \times G \rightarrow \mathscr{U}$ such that $\alpha$ is strongly Borel (with respect to $d g \otimes d g$ ) and $T(x) a$ is a strongly Borel map from $G$ to $A$ for each $a \in A$. Moreover, we demand

(1) $[T(x) \alpha(y, z)] \alpha(x, y z)=\alpha(x, y) \alpha(x y, z)$,

(2) $[T(x) T(y) a] \alpha(x, y)=\alpha(x, y)[T(x y) a]$,

(3) $\alpha(x, e)=\alpha(e, y)=1, T(e)=1$

for all $x, y, z \in G$ and $a \in A$, with $e$ being the identity in $G$. (The equation (1) will be familiar as the usual cocycle identity.)

THEOREM 2.2. Let $(T, \alpha)$ be a twisting pair for $G$ and $A$. If we define a product, $\cdot$, and an involution, *, on the Banach space of all Bochner-integrable functions from $G$ to $A$ by

$$
(f \cdot h)(x)=\int_{G} f(y)\left[T(y) h\left(y^{-1} x\right)\right] \alpha\left(y, y^{-1} x\right) d y
$$

and, writing $\Delta_{G}$ for the modular function on $G$,

$$
f^{*}(x)=\alpha\left(x, x^{-1}\right)^{*}\left[T(x) f\left(x^{-1}\right)^{*}\right] \Delta_{G}\left(x^{-1}\right)
$$

the result is a Banach*-algebra which we denote $L^{1}(A, G ; T, \alpha)$, the twisted group algebra on $G$ with values in $A$ produced by the twisting pair $(T, \alpha)$.

Proof. The required computations are carried out in $\$ 6$.

Let Aut $_{1}(A)$ and $\mathscr{U}$ be as previously defined and let $B \subset$ Aut $_{1}(A)$ be the set of all automorphisms of $A$ of the form $I_{u}, u \in \mathscr{U}$, where for any $a \in A, I_{u}(a)=u a u^{*}$. $B$ is clearly a subgroup of $\operatorname{Aut}_{1}(A)$ and it is normal since $u \in \mathscr{U}$ and $z \in \operatorname{Aut}_{1}(A)$ imply that $z^{-1} I_{u} z=I_{z^{-1}{ }_{(u)}}$ (again the unique extension of $z$ to Aut $(M(A)$ ) is also denoted by $z$ ). If $\operatorname{Aut}_{1}(A)$ is given the strong topology, $B$ will be a closed normal subgroup. We let $p:$ Aut $_{1}(A) \rightarrow$ Aut $_{1}(A) / B$ be the natural projection, which will be continuous of course if $\operatorname{Aut}_{1}(A) / B$ is given the quotient topology.

Proposition 2.1.a. With notations as above we have:

$$
p T: G \rightarrow \operatorname{Aut}_{1}(A) / B \text { is a continuous homomorphism. }
$$

Proof. It is easy to show that since $\operatorname{Aut}_{1}(A)$ is a closed subset of the unit sphere of $B(A, A)$ (the bounded linear operators on $A$ ), and $A$ is separable, $\operatorname{Aut}_{1}(A)$ (and thus also $\operatorname{Aut}_{1}(A) / B$ ) is strongly metrizable. Also $G$ is a polish group since it is 
second-countable and locally compact. Since $p T$ is a Borel homomorphism, the proposition follows from a Theorem of Banach (to be found for example on p. 25 of his book Théorie des opérations linéaires).

To indicate the scope of Definition 2.1 we give some examples of twisted group algebras.

EXAMPLE 1. The usual complex group algebra $L^{1}(G)$ is a twisted group algebra with $A=C$ and the twist trivial (i.e. $T$ and $\alpha$ the identities).

EXAMPLE 2. The completion of the usual algebra tensor product of $A$ with $L^{1}(G)$, completed with respect to the greatest cross-norm $\gamma, A \otimes_{\gamma} L^{1}(G)$, is a twisted group algebra with the twist trivial.

EXAMPLE 3. Let $G$ be a locally compact transformation group acting on a locally compact space $X$ with the group action denoted by $x \rightarrow g x$. Let $A$ be the algebra of all continuous functions on $X$ vanishing at infinity, let $\alpha$ be trivial, and define $T$ by $[T(g) f](x)=f\left(g^{-1} x\right)$.

ExAMPLE 4. Let $G$ be any locally compact group and let $A$ be the complex numbers. Then $\alpha$ must be complex valued and $T$ must be trivial. We call this algebra $L_{1}(G ; \alpha)$.

EXAMPLE 5. A very important class of examples arises when $\alpha$ is trivial and $A$ is a $C^{*}$-algebra. The resulting twisted group algebra has representations which appear to be important to quantum physics. In particular if $G$ is a group of automorphisms of $A$, these representations have been studied under the name of covariant representations of $(G, A)$ by several authors.

EXAMPLE 6. Suppose $0 \rightarrow H \stackrel{\sigma}{\rightarrow} N \stackrel{\pi}{\rightarrow} K \rightarrow 0$ is an exact sequence of locally compact groups. Then $\pi$ has a Borel measurable right inverse, $\eta$. (For very general circumstances under which this happens, see [7].) We define a twisting pair $(T, \alpha)$ such that $L^{1}\left(L^{1}(H), K ; T, \alpha\right)$ is isometrically isomorphic with $L^{1}(N)$.

Let $\Delta$ be the modular function for left Haar measure $d h$ on $H$. We identify $H$ and $\sigma(H)$. For $k \in K, h \in H$, define

$$
h^{k}=\eta(k)^{-1} h \eta(k) ; \quad h_{k}=\eta(k) h \eta(k)^{-1} .
$$

By the uniqueness of Haar measure there are positive real numbers $\delta(k)$ such that $\delta(k) d h_{k}=d h$. Writing $\beta\left(k_{1}, k_{2}\right)=\eta\left(k_{1}\right) \eta\left(k_{2}\right) \eta\left(k_{1} k_{2}\right)^{-1}$, for $k_{1}, k_{2} \in K$, we define a twisting pair $(T, \alpha)$ by

$$
\begin{aligned}
(T(k) f)(h) & =\delta(k)^{-1} f\left(h^{k}\right), \\
\left(\alpha\left(k_{1}, k_{2}\right) f\right)(h) & =f\left(\beta\left(k_{1}, k_{2}\right)^{-1} h\right) \\
\left(f \alpha\left(k_{1}, k_{2}\right)\right)(h) & =\Delta\left(\beta\left(k_{1}, k_{2}\right)\right)^{-1} f\left(h \beta\left(k_{1}, k_{2}\right)^{-1}\right)
\end{aligned}
$$

for $k, k_{1}, k_{2} \in K, f \in L^{1}(H), h \in H$. Note that $\beta\left(k_{1}, k_{2}\right) \in H$. In $\S 6$ we exhibit the computations which demonstrate that $(T, \alpha)$ is a twisting pair producing a twisted group algebra on $K$ with values in $L^{1}(H)$ and that $L^{1}\left(L^{1}(H), K ; T, \alpha\right)$ is isometrically isomorphic with $L^{1}(N)$.

In connection with this example we state the following converse result. 
THeORem 2.3. Suppose $H$ and $K$ are locally compact groups, and $(T, \alpha)$ is a twisting pair for $\left(K, L^{1}(H)\right)$. Then $\alpha$ can be written as $\hat{\alpha} \beta$ where $(T, \beta)$ is a twisting pair for $\left(K, L^{1}(H)\right)$ and $\hat{\alpha}$ is a complex valued cocycle, and there is a locally compact group extension $G$ of $K$ by $H$ such that:

(1) $L^{1}\left(L^{1}(H), K ; T, \beta\right)$ is isomorphic with $L^{1}(G)$ in the manner discussed in Example 6 and

(2) $L^{1}\left(L^{1}(H), K ; T, \alpha\right)$ is isomorphic with the algebra $L^{1}(G ; \hat{\alpha})$ discussed as Example 4. Here we are considering the cocycle $\hat{\alpha}$ on $K$ as a cocycle on $G$ constant on cosets.

Proof. We defer this to $\S 6$.

The trivial case of Example 1 is, of course, classical. Example 2 was studied by G. P. Johnson in [12]. The algebras of Example 3 have been considered from a rather different point of view, attention being restricted to the enveloping $C^{*}$ algebras, by Glimm [8] and by Effros and Hahn [6].

The algebras of Example 4 have been defined and studied briefly by L. Auslander and C. C. Moore in [2]. Example 6 has been one of our primary motivations, and a guide in formulating twisted group algebras. In a rather different formulation, Leptin has studied this example for the case where the group $H$ is abelian and $T$ is a homomorphism. Some of our terminology arises directly from this example. For instance, when $\alpha$ is trivial, we speak of the "split case" since it corresponds to the case where the exact sequence splits in this example and $N$ is a semidirect product. Likewise, our "induced representations" correspond to induced representations of $N$ in the sense of Mackey [17].

We now show that the algebra $L^{1}(A, G ; T, \alpha)$ depends only on an equivalence class of the twisting pair $(T, \alpha)$.

Definition 2.4. Let $A$ be a Banach*-algebra with approximate identity, and let $G$ be a locally compact group. We will denote by $B(G, A)$ the group of all Borel functions $p$ from $G$ to $\mathscr{U}(M(A)$ ) (the unitary double centralizers of $A$ ) such that $p(e)=1$, with pointwise operations, and by $Z(G, A)$ the set of all twisting pairs for $A, G$.

Let $P \in B(G, A)$ and let $(T, \alpha) \in Z(G, A)$. Let $(T, \alpha)_{p}=\left(T_{p}, \alpha_{p}\right)$ where for $x, y \in G$, $a \in A$, we define $T_{p}$ and $\alpha_{p}$ by:

1. $T_{p}(x) a=p(x)(T(x) a) p(x)^{*}$,

2. $\alpha_{p}(x, y)=p(x)[T(x) p(y)] \alpha(x, y) p(x y)^{*}$.

Proposition 2.5. With notation as above, $(T, \alpha)_{p} \in Z(G, A)$.

Proof. Measurability considerations are immediate. We verify the first identity. Let $x, y, z \in G$. Then:

$$
\begin{aligned}
T_{p}(x) \alpha_{p}(y, z) & =p(x) T(x)\left[p(y)[T(y) p(z)] \alpha(y, z) p(y z)^{*}\right] p(x)^{*} \\
& =p(x)[T(x) p(y)][T(x) T(y) p(z)][T(x) \alpha(y, z)]\left[T(x) p(y z)^{*}\right] p(x)^{*} .
\end{aligned}
$$


Also $\alpha_{p}(x, y z)=p(x)[T(x) p(y z)] \alpha(x, y z) p(x y z)^{*}$. Therefore

$$
\begin{aligned}
T_{p}(x) \alpha_{p}(y, z) \alpha_{p}(x, y z) & =p(x)[T(x) p(y)][T(x) T(y) p(z)] \alpha(x, y) \alpha(x y, z) p(x y z)^{*} \\
& =p(x)[T(x) p(y)] \alpha(x, y)[T(x y) p(z)] \alpha(x y, z) p(x y z)^{*} \\
& =\left[\alpha_{p}(x, y) p(x y)\right][T(x y) p(z)] \alpha(x y, z) p(x y z)^{*} \\
& =\alpha_{p}(x, y) \alpha_{p}(x y, z) .
\end{aligned}
$$

The second identity is verified in a similar way.

Proposition 2.6. The map $(p,(T, \alpha)) \rightarrow(T, \alpha)_{p}$ from $B(G, A) \times Z(G, A) \rightarrow$ $Z(G, A)$ defines an action of $B(G, A)$ as a transformation group on the $Z(G, A)$.

Proof. Clearly $(T, \alpha)_{1}=(T, \alpha)$ where 1 is the function identically equal to 1 . We show that if $p, q \in B(G, A)$, and $(T, \alpha) \in Z(G, A),\left[(T, \alpha)_{q}\right]_{p}=(T, \alpha)_{p q}$.

It is immediate that $\left(T_{q}\right)_{p}=T_{p q}$.

$$
\begin{aligned}
\left(\alpha_{q}\right)_{p}(x, y) & =p(x)\left[T_{q}(x) p(y)\right] \alpha_{q}(x, y) p(x y)^{*} \\
& =p(x) q(x)[T(x) p(y)][T(x) q(y)] \alpha(x, y) q(x y)^{*} p(x y)^{*} \\
& =\alpha_{p q}(x, y) .
\end{aligned}
$$

This completes the proof.

Denote the quotient set $Z(G, A) / B(G, A)$ by $H(G, A)$.

THEOREM 2.7. If $(T, \alpha)$ is a twisting pair for $(A, G)$ the isomorphism class of $L^{1}(A, G ; T, \alpha)$ depends only on the equivalence class of $(T, \alpha)$ in $H(G, A)$.

Proof. We will show that $L^{1}(A, G ; T, \alpha)$ and $L^{1}\left(A, G ; T_{p}, \alpha_{p}\right)$ are isometrically *-isomorphic for any $p \in B(G, A)$.

Let $f \in L^{1}(A, G ; T, \alpha)$ and define $\Phi_{p}(f)$ by $\Phi(f)(x)=f(x) p(x)^{*}$. We claim that $\Phi_{p}$ is the desired isomorphism. It is obviously a linear isometry from $L^{1}(A, G ; T, \alpha)$ to $L^{1}\left(A, G ; T_{p}, \alpha_{p}\right)$. If $f, g \in L^{1}(A, G ; T, \alpha)$ :

$$
\begin{aligned}
{[\Phi(f) \cdot \Phi(g)](x) } & =\int_{G} \Phi(f)(y)\left[T_{p}(y)\left(\Phi(g)\left(y^{-1} x\right)\right)\right] \alpha_{p}\left(y, y^{-1} x\right) d y \\
& =\int_{G} f(y) p(y)^{*} p(y) T(y)\left[g\left(y^{-1} x\right) p\left(y^{-1} x\right)^{*}\right] \\
\cdot p(y)^{*} p(y)\left[T(y) p\left(y^{-1} x\right)\right] \alpha\left(y, y^{-1} x\right) p(x)^{*} d y & \\
& =\int f(y)\left[T(y) g\left(y^{-1} x\right)\right] \alpha\left(y, y^{-1} x\right) d y p(x)^{*} \\
& =\Phi(f \cdot g)(x) .
\end{aligned}
$$

In a similar way we show that $\Phi$ preserves involution, and this completes the proof.

If $A$ is abelian and $(T, \alpha) \in Z(G, A), T$ is a homomorphism and the set $Z_{T}(G, A)$ $=\{(S, \alpha) \in Z(G, A) \mid S=T\}$ forms a group under pointwise multiplication of the "cocycles" $\alpha$. If $p \in B(G, A)$ and $\hat{p}: G \times G \rightarrow A$ is defined by

$$
\hat{p}(x, y)=T(x) p(y) p(x y)^{-1} p(x),
$$


then $\hat{p} \in Z_{T}(G, A)$, ( $\hat{p}$ is usually called the "coboundary" of $p$ ). In this way $B(G, A)$ maps into a subgroup $\hat{B}(G, A)$ of $Z_{T}(G, A)$. It is easy to see that the group $Z_{T}(G, A) / \hat{B}(G, A)=H_{T}(G, A)$ is precisely the set of orbits of $Z_{T}(G, A)$ under the action of $\hat{B}(G, A)$. Thus in the case of commutative $A, H(G, A)$ is the union of the usual "cohomology groups" $H_{T}(G, A)$.

3. Representations of twisted group algebras. In this section we discuss general results concerning representations of $L^{1}(A, G ; T, \alpha)$ for a fixed $A, G, T$ and $\alpha$. The Banach algebra $A$ will always be assumed to be a *-algebra with a two-sided approximate identity of unit norm. Representation will always mean continuous *-representation by bounded operators on a Hilbert space. We will need the idea of the left centralizer algebra of a Banach *-algebra $A$, which is also developed in [11]. A left centralizer on $A$ is a continuous linear map $m: A \rightarrow A$ such that $m(x y)=(m x) y$ for all $x, y$ in $A$. We will let $M(A)_{L}$ be the Banach *-algebra of left centralizers on $A$. It is shown in [11] that we always have $M(A) \subset M(A)_{L}$. It is also shown that, just as in the case of double centralizers, automorphisms of $A$ lift uniquely to automorphisms of $M(A)_{L}$.

Lemma 3.1. (a) The Banach *-algebra $M(A)$, the double centralizer algebra of $A$, has an isometric *-isomorphic embedding in $M\left(L^{1}(A, G ; T, \alpha)\right)_{L}$ given by $(m \cdot f) x$ $=m(f(x))$; where $m \in M(A), f \in L^{1}(A, G ; T, \alpha)$.

(b) The set of regular Borel measures with compact support is embedded in $M\left(L^{1}(A, G ; T, \alpha)\right)_{L} b y$

$$
(\mu \cdot f)(x)=\int_{G}\left[T(y) f\left(y^{-1} x\right)\right] \alpha\left(y, y^{-1} x\right) d \mu(y) .
$$

(c) If we use $x \cdot f$ to denote the unit point measure at $x \in G$ acting on $f$ as in (b), then

$$
x \cdot(y \cdot f)=\alpha(x, y) \cdot(x y \cdot f)
$$

for all $x, y \in G, f \in L^{1}(A, G ; T, \alpha)$. Moreover, for $m \in M(A), x \in G$,

$$
T(x) m \cdot(x \cdot f)=x \cdot(m \cdot f) .
$$

Proof. (a) Let $f$ and $g$ be in $L^{1}(A, G ; T, \alpha)$, and $m \in M(A)_{L}$. Then

$$
(m \cdot f) \cdot g(x)=\int_{G}(m \cdot f)(y)\left[T(y) g\left(y^{-1} x\right)\right] \alpha\left(y, y^{-1} x\right) d y=m \cdot(f \cdot g)(x) .
$$

Thus $m$ does become a left centralizer of $L^{1}(A, G ; T, \alpha)$. We will not normally distinguish $m$ from its image under this embedding. It is clear that this embedding preserves products and involutions. (See [11] for definitions of these in the double centralizer algebra.) Clearly the norm of $m \cdot f$ cannot exceed the norm of $m$ acting on $A$ times the norm of $f$, so the embedding is not norm increasing. On the other hand, taking $f$ to be $a \otimes \varphi$ for some $\varphi \in L^{1}(G)$ and all $a \in A$, we see that the embedding cannot be norm decreasing, so it is isometric. 
(b) Let $\mu$ be a regular Borel measure on $G$ with compact support. Then

$$
\begin{aligned}
\mu \cdot(f \cdot g)(x) & =\int_{G}\left[T(y) f \cdot g\left(y^{-1} x\right)\right] \alpha\left(y, y^{-1} x\right) d \mu(y) \\
& =\int_{G}\left\{T(y)\left[\int_{G} f(z)\left[T(z) g\left(z^{-1} y^{-1} x\right)\right] \alpha\left(z, z^{-1} y^{-1} x\right)\right]\right\} \alpha\left(y, y^{-1} x\right) d z d \mu(y)
\end{aligned}
$$

(by using the appropriate identities concerning $T$ and $\alpha$ )

$$
=\int_{G} \int_{G}[T(y) f(z)]\left[\alpha(y, z)\left[T(y z) g\left(z^{-1} y^{-1} x\right)\right] \alpha\left(y z, z^{-1} y^{-1} x\right)\right] d z d \mu(y) .
$$

By changing the order of integration and substituting, we see that the above integral is equal to

$$
\begin{aligned}
& \int_{G} \int_{G}\left[T(z) f\left(z^{-1} y\right)\right] \alpha\left(z, z^{-1} y\right)\left[T(y) g\left(y^{-1} x\right)\right] \alpha\left(y, y^{-1} x\right) d \mu(z) d y \\
& =\int_{G}(\mu \cdot f)(y)\left[T(y) g\left(y^{-1} x\right)\right] \alpha\left(y, y^{-1} x\right) d y=((\mu \cdot f) \cdot g)(x) . \\
& \begin{aligned}
\text { (c) } \quad[x \cdot(y \cdot f)](z) & =\left[T(x)(y \cdot f)\left(x^{-1} f\right)\right] \alpha\left(x, x^{-1} z\right) \\
& =\left[T(x)\left\{\left[T(y) f\left(y^{-1} x^{-1} z\right)\right] \alpha\left(y, y^{-1} x^{-1} z\right)\right\}\right] \alpha\left(x, x^{-1} z\right) \\
& =\left[T(x) T(y) f\left(y^{-1} x^{-1} z\right)\right] \alpha(x, y) \alpha\left(x y, y^{-1} x^{-1} z\right) \\
& =\alpha(x, y)\left[T(x y) f\left((x y)^{-1} z\right)\right] \alpha\left(x y, y^{-1} x^{-1} z\right) \\
& =\alpha(x, y)(x y \cdot f(z))=\alpha(x, y) \cdot(x y \cdot f)(z) .
\end{aligned}
\end{aligned}
$$

Now

$$
\begin{aligned}
(T(x) m \cdot(x \cdot f))(y) & =T(x) m(x \cdot f(y)) \\
& =T(x) m T(x)\left(f\left(x^{-1} y\right)\right) \alpha\left(x, x^{-1} y\right) \\
& =T(x)\left(m \cdot f\left(x^{-1} y\right)\right) \alpha\left(x, x^{-1} y\right) \\
& =x \cdot(m \cdot f)(y) .
\end{aligned}
$$

This completes (c).

B. E. Johnson in [11] has shown that if $\pi$ is an essential representation of any Banach *-algebra $B$ with norm bounded left approximate identity on a Hilbert space $H$, then $\pi$ extends uniquely to $M(B)_{L}$. The extension will also be denoted by $\pi$ and can be explicitly constructed as follows: If $\xi=\sum_{i=1}^{n} \pi\left(b_{i}\right) \zeta_{i}, \zeta_{i} \in H, b_{i} \in B$, and if $m \in M(B)_{L}$ then $\pi(m) \xi=\sum_{i=1}^{n} \pi\left(m b_{i}\right) \xi_{i}$ defines $\pi(m)$ as a linear operator on the linear span of $\pi(B)(H)$. This operator extends to a bounded linear operator on $H$ and $m \rightarrow \pi(m)$ is a representation of $M(B)_{L}$. Moreover $\pi\left(M(B)_{L}\right)$ is in the weak operator closure of $\pi(B)$. For the proofs of all these results see [11].

In order to use the above result in our context, we will need to have a norm bounded left approximate identity in $L^{1}(A, G ; T, \alpha)$. We conjecture that there is always such an approximate identity, but examples show that it is not always the 
most obvious candidate, namely the tensor product of approximate identities in $A$ and $L^{1}(G)$. The following result gives a sufficient condition.

THEOREM 3.2. Let $\left(F_{i}\right)_{i \in I}$ be the approximate identity in $L^{1}(G)$ formed from the normalized characteristic functions of the neighborhoods of $e$ in $G$. Suppose $A$ has a countable approximate identity $\left(a_{n}\right), 1 \leqq n \leqq \infty$ and suppose $x \rightarrow x \cdot f$ (notation as before $)$ is continuous at e for all $f \in L^{1}(A, G ; T, \alpha)$ then the set $\left\{F_{i} \otimes a_{n}\right\}$ is a left approximate identity of norm one.

Proof. For $f \in L^{1}(A, G ; T, \alpha)$,

$$
\begin{aligned}
\left\|\left(F_{i} \otimes a_{n}\right) f-f\right\| & \leqq \int_{G} \int_{G}\left\|F_{i}(y) a_{n}\left(T(y) f\left(y^{-1} x\right)\right) \alpha\left(y, y^{-1} x\right)-f(x)\right\| d x d y \\
& =\mu\left(S_{i}\right)^{-1} \int_{S_{i}} \int_{G}\left\|a_{n}\left(T(y) f\left(y^{-1} x\right)\right) \alpha\left(y, y^{-1} x\right)-f(x)\right\| d x d y
\end{aligned}
$$

$\left(F_{i}=\mu\left(S_{i}\right)^{-1} \chi_{S_{i}}\right.$ for some neighborhood $S_{i}$ of $e$ ). By the Lebesgue dominated convergence theorem, this expression converges to $\mu\left(S_{i}\right)^{-1} \int_{S_{i}}\|y \cdot f-f\| d y$ as $n \rightarrow \infty$, and by assumption this converges to zero on $i$. The condition given in the theorem is frequently easy to verify. It is certainly true, for example, if $T$ and $\alpha$ are weakly continuous (e.g. if $G$ is discrete), or if $\alpha$ is trivial and $T$ is weakly continuous at $e$. In the case of the pair arising from a group extension, the condition is equivalent with the existence of a local measurable cross-section about the identity, continuous at the identity.

Definition. Let $G$ be a locally compact group, $A$ a Banach *-algebra with approximate identity, and $(T, \alpha)$ a twisting pair for $A$ and $G$. By a representing pair for the 4-tuple $(A, G, T, \alpha)$ we will mean a pair $(\pi, U)$ where:

(1) $\pi$ is a nondegenerate representation of $A$ on $H$.

(2) $U$ is a weakly measurable mapping from $G$ to $\mathscr{U}(H)$, the unitary operators on $H$, such that $U_{x} U_{y}=\pi(\alpha(x, y)) U_{x y}, \forall x, y \in G$.

(3) $U_{x} \pi(a) U_{x}^{*}=\pi(T(x) a)$ for all $x \in G, a \in A$.

If $G$ is any locally compact group, $H$ is a Hilbert space, $\mathscr{U}(H)$ denotes the group of unitaries on $H$, and $K$ is a subgroup of $\mathscr{U}(H)$, and if $\nu: \mathscr{U}(H) \rightarrow \mathscr{U}(H) / K$ is the natural projection, we will define a generalized $K$-projective representation of $G$ on $H$ to be a weakly measurable mapping $\rho$ of $G$ into $\mathscr{U}(H)$ such that $\nu \rho$ is a group homomorphism. This definition generalizes the usual definition of projective representation and has been investigated by Leptin in [14]. Condition (2) in the definition of representing pair implies that $U$ is a $\pi(\mathscr{U})$-projective representation of $G$ on $H$, where $\mathscr{U}$ is the group of unitaries in $M(A)$.

ThEOREM 3.3. Suppose $L^{1}(A, G ; T, \alpha)$ has a norm bounded left approximate identity. Then:

(1) There is a one-to-one correspondence between representing pairs for $(A, G, T, \alpha)$ and nondegenerate representations of $L^{1}(A, G ; T, \alpha)$. 
(2) If $(\pi, U)$ is a representing pair for $(A, G, T, \alpha)$, the corresponding representation $\pi$ of $L^{1}(A, G, T, \alpha)$ is given by:

$$
\Pi(f)=\int_{G} \pi(f(x)) U(x) d x .
$$

$\Pi$ will be called the integrated form of $(\pi, U)$.

(3) $\Pi\left(L^{1}(A, G ; T, \alpha)\right)$ and the set $\pi(A) \cup U(G)$ generate the same von Neumann algebra on $H$.

Proof. Let $(\pi, U)$ be a representing pair for $(A, G, T, \alpha)$. Construct $\Pi$ as in (r). We first show that $\Pi$ is a representation.

$$
\begin{aligned}
\Pi(f \cdot g) & =\int_{G} \int_{G} \pi(f(y)) \pi\left(T(y) g\left(y^{-1} x\right)\right) \pi\left(\alpha\left(y, y^{-1} x\right)\right) U(x) d y d x \\
& =\int_{G} \int_{G} \pi(f(y)) U(y) \pi\left(g\left(y^{-1} x\right)\right) U(y)^{*} \pi\left(\alpha\left(y, y^{-1} x\right)\right) U(x) d x d y \\
& =\int_{G} \int_{G} \pi(f(y)) U(y) \pi\left(g\left(y^{-1} x\right)\right) U\left(y^{-1} x\right) d x d y \\
& =\int_{G} \pi(f(y)) U(y) d y \int_{G} \pi(g(y)) U(y) d y \\
& =\Pi(f) \Pi(g) .
\end{aligned}
$$

Now let $\xi, \eta \in H$. We have

$$
\begin{aligned}
\left(\Pi\left(f^{*}\right) \xi \mid \eta\right) & =\int_{G}\left(\pi f^{*}(x) U(x) \xi \mid \eta\right) d x \\
& =\int_{G}\left(\Delta\left(x^{-1}\right) \pi\left(\alpha\left(x, x^{-1}\right)^{*}\right) \pi\left(T(x) f\left(x^{-1}\right)\right)^{*} U(x) \xi \mid \eta\right) d x \\
& =\int_{G}\left(\Delta\left(x^{-1}\right) \pi\left(\alpha\left(x, x^{-1}\right)^{*}\right) U(x) \pi\left(f\left(x^{-1}\right)\right)^{*} \xi \mid \eta\right) d x \\
& =\int_{G}\left(\Delta\left(x^{-1}\right) U\left(x^{-1}\right)^{*} \pi\left(f\left(x^{-1}\right)\right)^{*} \xi \mid \eta\right) d x \\
& =\int_{G}\left([\pi(f(x)) U(x)]^{*} \xi \mid \eta\right) d x=\int_{G}(\xi \mid \pi(f(x)) U(x) \eta) d x \\
& =(\xi \mid \Pi(f) \eta)=\left(\Pi(f)^{*} \xi \mid \eta\right) .
\end{aligned}
$$

Thus $\Pi$ preserves *. If $f_{\mathfrak{i}}$ is an approximate identity in $L^{1}(A, G ; T, \alpha) \xi \in H$ and 
$a \in A \subset M(A)$, then $\Pi\left(a \cdot f_{i}\right) \xi \rightarrow \pi(a) \xi$ and since $\pi(A)(H)$ is dense in $H$, so is $\Pi\left(L^{1}(A, G ; T, \alpha)\right) H$. Thus $\Pi$ is nondegenerate.

Now suppose $\Pi$ is a representation of $L^{1}(A, G ; T, \alpha)$. Since both $G$ and $A$ can be embedded in $M\left(L^{1}(A, G ; T, \alpha)\right)_{L}$, we may extend $\Pi$ to both $G$ and $A$. The extensions may be denoted by $U$ and $\pi$ respectively and by previously cited results are explicitly given by:

$$
\pi(a)\left(\sum_{i=1}^{n} \Pi\left(f_{i}\right) \xi_{i}\right)=\Pi\left(\sum_{i=1}^{n}\left(a \cdot f_{i}\right) \xi_{i}\right)
$$

and

$$
U(x)\left(\sum_{i=1}^{n} \Pi\left(f_{i}\right) \xi_{i}\right)=\Pi\left(\sum_{i=1}^{n}(x \cdot f) \xi_{i}\right)
$$

on the linear span of $\Pi\left(L^{1}(A, G ; T, \alpha)\right) H$. Since $A \subset M(A)$ is *-homomorphically and continuously embedded in $M\left(L^{1}(A, G ; T, \alpha)\right)_{L}, \pi$ is a representation of $A$.

Now $G$ is embedded in $M\left(L^{1}(A, G ; T, \alpha)\right)_{L}$ as a set of unitaries, as is easily seen. Thus $U(x)$ is a unitary for all $x \in G$. For $x, y \in G$ and $f \in L^{1}(A, G ; T, \alpha)$ we have:

$$
\begin{aligned}
U(x) U(y)\left(\sum_{i=1}^{n} \Pi\left(f_{i}\right) \xi_{i}\right) & =\sum_{i=1}^{n} \Pi\left(x \cdot\left(y \cdot f_{i}\right)\right) \xi_{i} \\
& =\sum_{i=1}^{n} \Pi(\alpha(x, y) \cdot(x y \cdot f)) \quad(\text { by Lemma 3.1(c)) } \\
& =\pi(\alpha(x, y)) U(x y)\left(\sum_{i=1}^{n} \pi\left(f_{i}\right) \xi_{i}\right) .
\end{aligned}
$$

Thus $U(x) U(y)$ and $\pi(\alpha(x, y)) U(x y)$ agree on a dense subset of $H$ and so are equal. Similarly, the second half of 3.1 (c) can be used to prove that $\pi(T(x) a) U(x)=$ $U(x) \pi(a)$. We have thus constructed a representing pair for $(A, G, T, \alpha)$ from $\Pi$. We must now show that the correspondence between representations of $L^{1}(A, G ; T, \alpha)$ and representing pairs for $(A, G, T, \alpha)$ given above is bijective.

Let $(\pi, U)$ be a representing pair for $(A, G, T, \alpha)$, form $\Pi$ as above from $(\pi, U)$ and again form the pair $\left(\pi^{\prime}, U^{\prime}\right)$ from $\Pi$ as illustrated above. Let $f_{i} \in L^{1}(A, G ; T, \alpha)$, $\xi_{i} \in H, a \in A$. Then

$$
\begin{aligned}
\pi^{\prime}(a) \sum_{i=1}^{n} \Pi\left(f_{i}\right) \xi_{i} & =\sum_{i=1}^{n} \Pi\left(a \cdot f_{i}\right) \xi_{i} \\
& =\sum_{i=1}^{n} \int_{G} \pi(a) \pi\left(f_{i}(x)\right) U(x) \xi_{i} d x \\
& =\pi(a) \sum_{i=1}^{n} \Pi\left(f_{i}\right) \xi_{i}
\end{aligned}
$$


hence $\pi^{\prime}=\pi$. Similarly

$$
\begin{aligned}
U^{\prime}(x) \sum_{i=1}^{n} \Pi\left(f_{i}\right) \xi_{i} & =\sum_{i=1}^{n} \int_{G} \pi\left(T(x) f_{i}\left(x^{-1} y\right)\right) \pi\left(\alpha\left(x, x^{-1} y\right)\right) U(y) \xi_{i} d y \\
& =\sum_{i=1}^{n} \int_{G} \pi\left(T(x) f_{i}\left(x^{-1} y\right)\right) U(x) U\left(x^{-1} y\right) \xi_{i} d y \\
& =\sum_{i=1}^{n} \int_{G} U(x) \pi\left(f_{i}(y)\right) U(y) \xi_{i} d y \\
& =U(x) \sum_{i=1}^{n} \Pi\left(f_{i}\right) \xi_{i}, \quad \text { and so } U^{\prime}=U .
\end{aligned}
$$

Now suppose a representation $\Pi$ of $L^{1}(A, G, T, \alpha)$ is given. Form $(\pi, U)$ from $\Pi$ and $\Pi^{\prime}$ from $(\pi, U)$ in the usual way.

Then if $a_{i} \in A, \varphi_{i} \in L^{1}(G), f_{i} \in L^{1}(A, G ; T, \alpha), \xi_{i} \in H$, we have

$$
\begin{aligned}
\sum_{j=1}^{n} \Pi^{\prime}\left(\sum_{i=1}^{m} a_{i} \otimes \varphi_{i}\right) \Pi\left(f_{j}\right) \xi_{j} & =\sum_{j=1}^{n} \sum_{i=1}^{m} \int_{G} \pi\left(a_{i}\right) \varphi_{i}(x) U(x) \Pi\left(f_{j}\right) \xi_{j} d x \\
& =\sum_{j=1}^{n} \sum_{i=1}^{m} \int_{G} \Pi\left(\varphi_{i}(x) a_{i} \cdot\left(x \cdot f_{j}\right)\right) \xi_{j} d x .
\end{aligned}
$$

Now

$$
\begin{aligned}
\int_{G} \varphi_{i}(x) a_{i} \cdot\left(x \cdot f_{j}\right)(y) d x & =\int_{G} \varphi_{i}(x) a_{i}\left[T(x) f_{j}\left(x^{-1} y\right)\right] \alpha\left(x, x^{-1} y\right) d x \\
& =\left(\varphi_{i} \otimes a_{i}\right) \cdot f_{j}(y) .
\end{aligned}
$$

Hence

$$
\int_{G} \varphi_{i}(x) a_{i} \cdot\left(x \cdot f_{j}\right) d x=\left(\varphi_{i} \otimes a_{i}\right) \cdot f_{j}
$$

and so

$$
\begin{aligned}
\sum_{j=1}^{n} \Pi^{\prime}\left(\sum_{i=1}^{m} a_{i} \otimes \varphi_{i}\right) \Pi\left(f_{j}\right) \xi_{j} & =\sum_{j=1}^{n} \sum_{i=1}^{m} \Pi\left[\left(\varphi \otimes a_{i}\right) \cdot f_{j}\right] \xi_{j} \\
& =\sum_{j=1}^{n} \Pi\left(\sum_{i=1}^{m} a_{i} \otimes \varphi_{i}\right) \Pi\left(f_{j}\right) \xi_{j}
\end{aligned}
$$

Since elements of the form $\sum_{i=1}^{m} a_{i} \otimes f_{i}$ are dense in $L^{1}(A, G ; T, \alpha), \Pi^{\prime}=\Pi$ and the correspondence is bijective.

It only remains to verify the final assertion of the theorem. Since $G$ and $A$ are embedded in the algebra $M\left(L^{1}(A, G ; T, \alpha)\right)_{L}$, both $U(G)$ and $\pi(A)$ are in the weak closure of $\Pi\left(L^{1}(A, G ; T, \alpha)\right)$ by [11]. On the other hand,

$$
\Pi(f)=\int_{G} \pi(f(x)) U(x) d x
$$


is the weak limit of sums of the form $\sum_{i=1}^{n} \pi\left(f\left(x_{i}\right)\right) U\left(x_{i}\right) \mu\left(S_{i}\right)$ where $\mu$ is the Haar measure of a set and the $S_{i}$ are measurable sets of finite measure. Since each such sum is in the weak closure of the algebra generated by $U(G) \cup \pi(A)$, so is $\Pi(f)$. This completes the theorem.

This theorem was proved in the case of Example 3 by Glimm in [8] and for the case where $G$ is a discrete group and $A$ is a $C^{*}$-algebra it has been announced by Zeller-Meier in [26]. Representing pairs for $(A, G, T, \alpha)$ when $\alpha$ is trivial, $A$ is a $C^{*}$-algebra, and $T$ is weakly continuous (or even norm continuous) have been considered by a number of authors, mostly in connection with applications to theoretical physics. Such a pair has been called a covariant representation of $A$ and $G$ by some authors.

We also remark that if $G$ is a locally compact group, the well-known correspondence between representations of $L^{1}(G)$ and unitary representations of $G$ is a trivial special case of the above theorem.

In a certain sense the twisted group algebras $L^{1}(G, A ; T, \alpha)$ are generalized tensor products. It is reasonable then to ask when representations of such algebras are tensor products of representations. For nontrivial $T$, the question must be reformulated in order to achieve meaningful results, but for trivial $T$ we have the following result.

Proposition 3.4. If $L^{1}(G, A ; \alpha)$ represents a twisted group algebra with a bounded left approximate identity and with $T$ trivial, and if either $A$ or $G$ is type I (see [3]), then every irreducible representation of $L^{1}(G, A ; \alpha)$ is isomorphic with the tensor product of an irreducible representation of $A$ and a generalized projective irreducible representation of $G$.

Proof. The proof is identical with the proof of the proposition when $\alpha$ is also trivial. This in turn is an immediate special case of [3, 13.1.8].

If $A$ is a $C^{*}$-algebra or a von Neumann algebra, a more detailed discussion of representations is often possible. We will now show that to a certain extent the representation theory of $L^{1}(G, A ; T, \alpha)$ for any $A$ can be reduced to the case where $A$ is a $C^{*}$-algebra, and this case can be further reduced to the case where $A$ is a von Neumann algebra. We will need the following results. If $A$ is any Banach *-algebra with approximate identity, the enveloping $C^{*}$-algebra of $A$ is the algebra $\bar{A}$ formed by giving $A$ the seminorm $\|\cdot\|^{\prime}$, where $\|x\|^{\prime}=\sup \|\pi(x)\|$ ( $\pi$ running over all representations of $A$ ), factoring out the ideal of all $x$ with $\|x\|^{\prime}=0$, and completing. If $\tau: A \rightarrow \bar{A}$ is the natural *-homomorphism and $\rho$ is a representation of $A$ on $H$, there is a unique representation $\bar{\rho}$ of $\bar{A}$ on $H$ such that $\rho=\bar{\rho} \tau$, and the algebra $\bar{\rho}(\bar{A})$ is the $C^{*}$-algebra generated by $\rho(A)$ (see $\left.[3,2.7 .1]\right)$.

If $A$ is a $C^{*}$-algebra, the universal enveloping von Neumann algebra is the weak closure $B$ of the image of $A$ under the direct sum $\pi_{0}$ of all irreducible representations of $A$. It is canonically isomorphic with the bidual $A^{* *}$ of $A$ with the Arens multiplication (see [1]). This algebra has a number of interesting properties, including 
the fact that if $\rho$ is any representation of $A$ on $H$, there is a unique normal $\left({ }^{1}\right)$ representation $\bar{\rho}$ of $B$ on $H$ such that $\rho=\bar{\rho} \pi_{0}$ and $\bar{\rho}(B)$ is the weak closure of $\rho(A)$. Also if $\gamma: A \rightarrow A$ is an automorphism, there is exactly one normal automorphism $\bar{\gamma}: B \rightarrow B$ extending $\gamma$. Both $\bar{\gamma}$ and its inverse are $\sigma(B, A)$ continuous on the unit ball of $B$ (see $[3, \S 12])$.

Proposition 3.5. If $A$ is a Banach *-algebra with approximate identity, $G$ is a locally compact group and $(T, \alpha)$ is a twisting pair for $(A, G)$, there is a $C^{*}$-algebra $\bar{A}$ and a twisting pair $(\bar{T}, \bar{\alpha})$ for $\bar{A}, G$ such that:

(1) There is a canonical continuous *-homomorphism

$$
k: L^{1}(A, G ; T, \alpha) \rightarrow L^{1}(\bar{A}, G ; \bar{T}, \bar{\alpha}) .
$$

(2) If $L^{1}(A, G ; T, \alpha)$ has a norm bounded left approximate identity and if $\Pi$ is any representation of $L^{1}(A, G ; T, \alpha)$ on $H$, there is a unique representation $\bar{\Pi}$ of $L^{1}(\bar{A}, G ; T, \bar{\alpha})$ on $H$ such that $\Pi=\bar{\Pi} k$.

Proof. (1) Let $\bar{A}$ be the enveloping $C^{*}$-algebra of $A$. If $x \in G$, then $T(x)$, being isometric, permutes the set of all representations of $A$. Thus if $a \in A$,

$$
\|T(x) a\|^{\prime}=\sup _{\pi}\|\pi(T(x) a)\|=\sup _{\pi}\|\pi(a)\|=\|a\|^{\prime} .
$$

Thus $T(x)$ is $\|\cdot\|^{\prime}$ isonietric and so extends to an (isometric) automorphism $\bar{T}(x)$ of $\bar{A}$. Since every representation of $A$ is the restriction of a representation of $M(A)$ (the double centralizer algebra), $\|\cdot\|^{\prime}$ extends to $M(A)$ and the resulting completion is $\bar{M}(A)$. We can thus easily see that $\bar{A}$ is a closed, two-sided ideal in $\bar{M}(A)$, and multiplication provides a natural homomorphism $\eta: \bar{M}(A) \rightarrow M(\bar{A})$. If $\sigma: M(A) \rightarrow \bar{M}(A)$ is the natural map, and $\gamma=\eta \sigma$, we let $\bar{\alpha}=\gamma \alpha$. Since $\gamma$ is a *-homomorphism and both $\gamma$ and the map $T \rightarrow \bar{T}$ are weakly continuous, it is easy to show that $(\bar{T}, \bar{\alpha})$ satisfies all the conditions (algebraic and measure theoretic) necessary for it to be a twisting pair. Thus $L^{1}(\bar{A}, G ; \bar{T}, \bar{\alpha})$ exists and there is an obvious homomorphism from $L^{1}(A, G ; T, \alpha)$ to it.

(2) Since any representing pair $(\pi, U)$ for $(A, G ; T, \alpha)$ produces the pair $(\bar{\pi}, U)$ for $(\bar{A}, G ; \bar{T}, \bar{\alpha})$, where $\bar{\pi}$ is the natural extension to $\bar{A}$, it follows easily that the integrated form of $(\pi, U)$ factors through the integrated form of $(\bar{\pi}, \bar{U})$. Theorem 3.3 then gives the desired conclusion.

Proposition 3.6. (1) If $A$ is a $C^{*}$-algebra, $G$ a locally compact group and $(T, \alpha)$ a twisting pair for $(A, G)$, then there is a twisting pair $(\bar{T}, \bar{\alpha})$ for $(B, G)$ (where $B$ is the enveloping von Neumann algebra of $A$ ) and a natural *-homomorphism

$$
J: L^{1}(A, G ; T, \alpha) \rightarrow L^{1}(B, G ; \bar{T}, \bar{\alpha}) .
$$

( $\left.{ }^{1}\right) \bar{\rho}$ is normal if whenever $S$ is a bounded family of selfadjoint elements of $B, \sup _{x \in S} \bar{\rho}(x)$ $=\bar{\rho}\left(\sup _{x \in S} x\right)$. 
(2) If $L^{1}(A, G ; T, \alpha)$ has a norm bounded left approximate identity, every representation of $L^{1}(A, G ; T, \alpha)$ factors through a unique representation of $L^{1}(B, G ; \bar{T}, \bar{\alpha})$.

Proof. By previous remarks, $T$ extends uniquely to a mapping $\bar{T}$ from $G$ to Aut $(B)$. With notation as before let $\pi_{0}: A \rightarrow B$ be the natural homomorphism. Since $\pi_{0}$ is a representation, it extends to $M(A)$ in such a way that $\pi_{0}(M(A)) \subset B$. If we let $\bar{\alpha}=\pi_{0} \alpha$, it is easy to see that $(\bar{T}, \bar{\alpha})$ is a twisting pair for $(G, B)$. The rest of the proposition is demonstrated by using the universal property of $B$ and Theorem 3.3 as we did in Proposition 3.5.

4. Induced representations. Let $A$ be a Banach *-algebra with approximate identity, and let $G, T, \alpha$ be as usual. Let $\pi$ be a representation of $A$ on $H$ and form the space $\mathscr{L}^{2}(G, H, d g)$ of Borel measurable, square integrable functions. Let $L^{2}(G, H, d g)$ be the usual associated Hilbert space.

Define $\tilde{\pi}$ and $U^{\pi}$ on $\mathscr{L}^{2}(G, H, d g)$ by:

(1) $[\tilde{\pi}(a) f](g)=\pi(T(g) a) f(g)$ and

(2) $\left[U^{\pi}\left(g_{0}\right) f\right](g)=\pi\left(\alpha\left(g, g_{0}\right)\right) f\left(g g_{0}\right) \Delta\left(g_{0}\right)^{1 / 2}$

where $f \in \mathscr{L}^{2}(G, H, d g)$ and $\Delta$ is the modular function in $G$. $\tilde{\pi}$ and $U^{\pi}$ preserve null functions and so define uniquely a pair of operators in $L^{2}(G, H, d g)$ which we also denote by $\left(\tilde{\pi}, U^{\pi}\right)$. (Which operators are meant will be clear from the context.)

THEOREM 4.1. $\left(\tilde{\pi}, U^{\pi}\right)$ is a representing pair for $(A, G, T, \alpha)$.

Proof. Clearly $\tilde{\pi}$ is a representation and $U^{\pi}\left(g_{0}\right)$ is unitary for all $g_{0}$. We claim

(a) $U^{\pi}\left(g_{0}\right) \tilde{\pi}(a)=\tilde{\pi}\left(T\left(g_{0}\right) a\right) U^{\pi}\left(g_{0}\right)$ and

(b) $U^{\pi}\left(g_{0}\right) U^{\pi}\left(g_{1}\right)=\tilde{\pi}\left(\alpha\left(g_{0}, g_{1}\right)\right) U^{\pi}\left(g_{0} g_{1}\right)$.

In fact

$$
\begin{aligned}
{\left[U^{\pi}\left(g_{0}\right) \tilde{\pi}(a) f\right](g) } & =\pi\left(\alpha\left(g, g_{0}\right)\right) \pi\left(T\left(g g_{0}\right) a\right) f\left(g g_{0}\right) \Delta\left(g_{0}\right)^{1 / 2} \\
& =\pi\left(T(g) T\left(g_{0}\right) a\right) \pi\left(\alpha\left(g, g_{0}\right)\right) f\left(g g_{0}\right) \Delta\left(g_{0}\right)^{1 / 2} \\
& =\left[\tilde{\pi}\left(T\left(g_{0}\right) a\right) U^{\pi}\left(g_{0}\right) f\right](g),
\end{aligned}
$$

which shows (a), and

$$
\begin{aligned}
{\left[U^{\pi}\left(g_{0}\right) U^{\pi}\left(g_{1}\right) f\right](g) } & =\pi\left(\alpha\left(g, g_{0}\right)\right) \pi\left(\alpha\left(g g_{0}, g_{1}\right)\right) f\left(g g_{0} g_{1}\right) \Delta\left(g_{0} g_{1}\right)^{1 / 2} \\
& =\pi\left(T(g) \alpha\left(g_{0}, g_{1}\right)\right) \pi\left(\alpha\left(g, g_{0} g_{1}\right)\right) f\left(g g_{0} g_{1}\right) \Delta\left(g_{0} g_{1}\right)^{1 / 2} \\
& =\left[\tilde{\pi}\left(\alpha\left(g_{0}, g_{1}\right)\right) U^{\pi}\left(g_{0} g_{1}\right) f\right](g)
\end{aligned}
$$

which proves (b). ( $\left.\tilde{\pi}, U^{\pi}\right)$ will be called the right induced representing pair, and its integrated form will be called the right induced representation. We could analogously define the left induced representation which for discrete $G$ was studied by Zeller-Meier. Symmetry makes it unnecessary to do so. We choose the right representation since it corresponds to the usage of the term "induced" by Mackey, as indicated in Theorem 4.2 below.

Let $0 \rightarrow H \stackrel{\sigma}{\rightarrow} G \stackrel{\rho}{\rightarrow} K \rightarrow 0$ be an exact sequence of locally compact groups. 
As previously indicated $L^{1}(G)$ is isometrically isomorphic with $L^{1}\left(L^{1}(H), K ; T, \alpha\right)$ for suitably defined $T$ and $\alpha$. Let $\pi$ be a representation of $L^{1}(H)$ and let $\Pi$ be the induced representation considered as a representation of $L^{1}(G)$. As is well known $\pi$ and $\Pi$ correspond uniquely to unitary representations $u$ and $U$ of $H$ and $G$ respectively.

THEOREM 4.2. $U$ is equivalent with the representation induced from $u$ in Mackey's sense [17].

Proof. We defer this proof to $\S 6$.

Definition 4.3. If $A, G, T, \alpha$ are as usual and $\left(\pi_{1}, U_{1}\right)$ and $\left(\pi_{2}, U_{2}\right)$ are representing pairs for $(A, G, T, \alpha)$ on $H$, we will say the pairs are unitarily equivalent if there is a unitary $W$ on $H$ such that $\pi_{2}=W^{*} \pi_{1} W$ and $U_{2}=W^{*} U_{1} W$. For this to occur, it is clearly necessary and sufficient that the integrated forms of $\left(\pi_{1}, U_{1}\right)$ and $\left(\pi_{2}, U_{2}\right)$ are unitarily equivalent in the usual sense.

For any representation $\pi$ of $A$ and any $g \in G$, we will denote the composition of $\pi$ with $T(g)$ by $\pi_{g}$.

THEOREM 4.4. (a) If $\pi$ is a representation of $A$ and $g \in G,\left(\tilde{\pi}, U^{\pi}\right)$ and $\left(\tilde{\pi}_{g}, U^{\pi_{g}}\right)$ are unitarily equivalent.

(b) If $\pi$ and $\rho$ are equivalent (resp. quasi-equivalent; see [3, 5.3]) representations of $A$, then $\left(\tilde{\pi}, U^{\pi}\right)$ and $\left(\tilde{\rho}, U^{\rho}\right)$ are equivalent (resp. quasi-equivalent).

Proof. (a) Let $W_{g_{0}}\left(g_{0} \in G\right)$ be defined on $\mathscr{L}^{2}(G, H, d g)$ by: $\left[W_{g_{0}} f\right](g)=$ $\pi\left(\alpha\left(g_{0}, g\right)\right) f\left(g_{0} g\right)$. $W_{g_{0}}$ is unitary and it is shown just as in Theorem 4.1 that $W_{g_{0}} \tilde{\pi} W_{g_{0}}^{*}=\tilde{\pi}_{g_{0}}$. Also,

$$
\begin{aligned}
{\left[W_{g_{0}} U^{\pi}\left(g_{1}\right) f\right](g) } & =\pi\left(\alpha\left(g_{0}, g\right) \alpha\left(g_{0} g, g_{1}\right)\right) f\left(g_{0} g g_{1}\right) \Delta\left(g_{1}\right)^{1 / 2} \\
& =\pi\left(T\left(g_{0}\right) \alpha\left(g, g_{1}\right)\right) \pi\left(\alpha\left(g_{0}, g g_{1}\right)\right) f\left(g_{0} g g_{1}\right) \Delta\left(g_{1}\right)^{1 / 2} \\
& =\left[U^{\pi_{g_{0}}}\left(g_{1}\right) W_{g_{0}} f\right](g)
\end{aligned}
$$

which proves (a).

(b) If $\varphi$ is a spatial (resp. algebraic) isomorphism of $\pi(A)$ with $\rho(A)$ so that $\rho=\pi \varphi$, and if $\hat{\varphi}$ is defined by:

$[(\hat{\varphi} \tilde{\pi})(a) f](g)=\varphi(\pi(T(g) a)) f(g)$ and $\left[\left(\hat{\varphi} U g_{0}\right) f\right](g)=\varphi\left(\pi\left(\alpha\left(g, g_{0}\right)\right)\right) f\left(g g_{0}\right) \Delta\left(g_{0}\right)^{1 / 2}$, then $\hat{\varphi}$ provides the required unitary (resp. algebraic) equivalence between $\left(\tilde{\pi}, U^{\pi}\right)$ and $\left(\tilde{\rho}, U^{\rho}\right)$.

COROLlaRY 4.5. If $T$ acts on the dual (resp. quasi-dual) see [3, §3. 7] by composition, and if for any irreducible (resp. factor) representation $\pi$ of $A$, the equivalence (resp. quasi-equivalence) class of $\pi$ is denoted $[\pi]$, then the equivalence (resp. quasiequivalence) class of the induced representation $\Pi$ depends only on the orbit (under $T$ ) of $[\pi]$ in the dual $\hat{A}$ (resp. the quasi-dual $\hat{A}$ ). Denote this action by

$$
[\pi] \rightarrow[\pi]_{x} \equiv\left[\pi_{x}\right]
$$


LEMMA 4.6. (a) $G$ acts on $\hat{A}$ and $\hat{A}$ (through $T$ ) as a transformation group.

(b) $G$ acts on $\hat{A}$ as a topological transformation group.

Proof. (a) If $a \in A, x, y \in G$ then

$$
\left(\pi_{y}\right)_{x}(a)=\pi(T(x) T(y) a)=\pi(\alpha(x, y)) \pi_{x y}(a) \pi(\alpha(x, y))^{*} .
$$

Since $\pi(\alpha(x, y))$ is unitary, $\left[[\pi]_{y}\right]_{x}=[\pi]_{x y}$. Similar computations show that $[\pi]_{e}=[\pi]$ (with $e$ the identity in $G$ ), and we are done.

(b) Glimm has proved in [8] that the group of automorphisms of $A$ in the strong topology acts on $\hat{A}$ as a topological transformation group in the case where $A$ is a $C^{*}$-algebra. Evidently this action is trivial on the subgroup $B$ of inner automorphisms by unitary double centralizers, and so $\operatorname{Aut}^{1}(A) / B$ acts as a topological transformation group on $\hat{A}$. Thus so does $G$.

Corollary 4.7. (a) The stabilizer $G_{[\pi]}$ of $[\pi]$ in $G$ (all $x \in G$ with $\left.[\pi]_{x}=[\pi]\right)$ is a subgroup of $G$ for any $\pi$ in $\hat{A}$ or $\hat{A}$.

(b) If $[\pi]$ is closed in $\hat{A}$, then $G_{[\pi]}$ is a closed subgroup.

(c) If $\hat{A}$ is a $T_{0}$ space, then $G_{[\pi]}$ is closed.

Proof. Everything is clear but (c). This follows from Lemma 4.6(b) and a result of Blattner [29, Lemma 1].

We now investigate the weak closure and commutant of an induced representation with an eye to determining when it is irreducible. From now until the end of this section, $(T, \alpha)$ will be a fixed twisting pair for $(G, A)$, and $\pi$ will be a fixed representation of $A$ on a Hilbert space $H$.

Suppose that for some $x \in G, N \in B(H)$, and all $a \in A, N \pi_{x}(a)=\pi(a) N$. Define $[N]_{x} \in B\left(L^{2}(G, H, m)\right)$ ( $m$ Haar measure) by: $\left([N]_{x} f\right)(g)=N \pi(\alpha(x, g)) f(x g)$. Then

Proposition 4.8. $[N]_{x}$ commutes with $\tilde{\pi}$ and $U^{\pi}$.

Proof.

$$
\begin{aligned}
{[N]_{x} \tilde{\pi}(a) f(g) } & =N \pi(\alpha(x, g)) \pi(T(x g) a) f(x g) \\
& =N \pi(T(x) T(g) a) \pi(\alpha(x, g)) f(x g) \\
& =\pi(T(g) a) N \pi(\alpha(x, g)) f(x g)=\tilde{\pi}(a)[N]_{x} f(g)
\end{aligned}
$$

Also

$$
\begin{aligned}
{[N]_{x} U_{y}^{\pi} f(g) } & =N \pi(\alpha(x, g) \alpha(x g, y)) f(x g y) \Delta^{1 / 2}(y) \\
& =N \pi(T(x) \alpha(g, y) \alpha(x, g y)) f(x g y) \Delta^{1 / 2}(y) \\
& =\pi(\alpha(g, y)) N \pi(\alpha(x, g y)) f(x g y) \Delta^{1 / 2}(y)=U_{y}^{\pi}[N]_{x} f(g) .
\end{aligned}
$$

This completes the proposition.

If we let $S_{x}=\left\{[N]_{x} \mid N \pi_{x}(a)=\pi(a) N, a \in A\right\}$ then the above proposition says that $\bigcup_{x \in G} S_{x}$ is contained in the commutant $\left(\tilde{\pi}, U^{\pi}\right)^{\prime}$ of the pair $\left(\tilde{\pi}, U^{\pi}\right)$. In particu$\operatorname{lar} S_{e}$ is pointwise multiplication by the commutant of $\pi$. Thus 
COROLlaRY 4.9. There is a one-to-one mapping of $\pi(A)^{\prime}$ into $\left(\tilde{\pi}, U^{\pi}\right)^{\prime}$ given by $N \rightarrow[N]_{e}$. In particular if $\left(\tilde{\pi}, U^{\pi}\right)$ is irreducible, then so is $\pi$.

Now let $D=L^{\infty}(G, m)$. $D$ is the von Neumann algebra generated by characteristic functions, which if allowed to act by left multiplication on $L^{2}(G, H, m)$ would form a projection valued measure on $G$. It is then easy to see that $D$ is a sort of system of imprimitivity for $\left(\tilde{\pi}, U^{\pi}\right)$. We then have the following proposition which is similar to [18, Theorem 6.4].

Proposition 4.10. The mapping $N \rightarrow[N]_{e}$ from $\pi(A)^{\prime}$ to $\left(\tilde{\pi}, U^{\pi}\right)^{\prime} \cap D^{\prime}$ is a linear isomorphism.

Proof. The mapping is clearly linear and one-to-one, and each $N$ is in $D^{\prime}$. It is known (see [4, Chapter 2, no. 2, §5, Theorem 1]) that an operator is in $D^{\prime}$ if and only if it is decomposable, i.e. it is given by pointwise multiplication by a bounded measurable $B(H)$-valued function on $G(B(H)$ represents the bounded linear operators on $H$ ). Let $R: G \rightarrow B(H)$ be such a function. If the associated operator also commutes with $\tilde{\pi}$ and $U^{\pi}$, then first of all $R(x) \in \pi(A)^{\prime}$ for all $x$ in $G$. Furthermore,

and

$$
\begin{aligned}
U^{\pi}(y)(R f)(x) & =\pi(\alpha(x, y)) R(x y) f(x y) \Delta(y)^{1 / 2} \\
& =R(x y) \pi(\alpha(x, y)) f(x y) \Delta(y)^{1 / 2}
\end{aligned}
$$

$$
\left(R U^{\pi}(y) f\right)(x)=R(x) \pi(\alpha(x, y)) f(x y) \Delta(y)^{1 / 2} .
$$

From this we see that $R(x y)=R(x)$ almost everywhere in $x$ and $y$. Hence $R$ is almost everywhere a constant $\bar{R}$. It is clear that $\bar{R} \in \pi(A)^{\prime}$ and that $[\bar{R}]_{e}=R$. This completes the proof.

We remark that if $\pi$ is irreducible and the $\pi_{x}$ are mutually inequivalent, or if $\pi$ is arbitrary and the $\pi_{x}$ are mutually disjoint, the above proposition specializes precisely to Mackey's theorem mentioned above [18, Theorem 6.4] for representations induced from normal subgroups. We now turn our attention to the case when $\pi$ is irreducible and give a more detailed analysis. Throughout the rest of this section $\pi$ will always be assumed to be irreducible.

LEMMA 4.11. Let the stability subgroup $G_{[\pi]}$ of $\pi$ be closed in $G$.

Then $\tilde{\pi}$ is unitarily equivalent with a representation $\rho$ of $A$ on $L^{2}(G, H, m)$ such that:

1. $\rho(A)$ is an algebra of measurable fields of operators in the sense of [4, Chapter 2].

2. Each function $\rho(a), a \in A$, has each $x \in G_{[\pi}$ as period.

3. $\|\rho(a)(x)\|=\|\tilde{\pi}(a)(x)\|$ for all $a \in A, x \in G$.

Proof. Note first that our assumptions guarantee the existence of a measurable cross-section $s$ from $G / G_{[\pi]}$ to $G$ (see [7]). Also from [27, Lemma 3], it follows that there is a measurable cross-section $n$ from the group $R$ of inner automorphisms of the algebra $B(H)$ to the group $\mathscr{U}(H)$ of unitaries on $H$. 
If for $g \in G$ we let $g_{c}=s\left(r(g)\right.$ ) (where $r: G \rightarrow G / G_{[\pi]}$ is projection) and $g_{s}=g g_{c}^{-1}$, then both $g \rightarrow g_{s}$ and $g \rightarrow g_{c}$ are measurable from $G$ to $G$ and every $g \in G$ can be uniquely written $g=g_{s} g_{c}$. Also if $x \in G_{\pi}$ and $z(x)$ is the unique (since $\pi$ is irreducible) element of $R$ such that $z(x) \pi(a)=\pi_{x}(a)$ for all $a$ in $A$, let $U_{x} \in \mathscr{U}(H)$ be defined by $U_{x}=n(z(x))$. Then $x \rightarrow U_{x}$ is strongly measurable and $U_{x}^{*} \pi(a) U_{x}=\pi_{x}(a)$ for all $a$ in $A$. The same automorphism is produced by $U_{x y}$ and $U_{y} U_{x} \pi(\alpha(x, y))$.

Finally let the unitary operator $V$ on $L^{2}(G, H, m)$ be defined by:

$$
(V f)(g) \equiv V_{g} f(g)=U_{g_{s}} \pi\left(\alpha\left(g_{s}, g_{c}\right)\right) f(g) .
$$

A straightforward computation using the properties of $T, \alpha$, and the unitaries $U_{x}$, $x \in G_{[\pi]}$ shows that for any $a \in A, V \tilde{\pi}(a) V^{*}=\rho(a)$ where $\rho(a)$ is the operator field defined by: $\rho(a)(g)=\pi\left(T\left(g_{c}\right) a\right)$. The functions $\rho(a)$ are all measurable by our assumptions. We also have for each $g$ in $G$ :

$$
\|\rho(a)(g)\|=\left\|V_{g} \pi(T(g) a) V_{g}^{*}\right\|=\|\pi(T(g) a)\| .
$$

These remarks together with some obvious properties of $\rho$ show that parts 1 and 3 of this lemma hold. To prove 2, note that if $g \in G$ and $x \in G_{[\pi]},(x g)_{c}=g_{c}$ and so $\rho(a)(x g)=\rho(a)(g)$ and each $\rho(a)$ has $x$ as period. This completes the proof of the lemma.

It can be shown easily that $g \rightarrow\|\tilde{\pi}(a)(g)\|$ (and therefore $g \rightarrow\|\rho(a)(g)\|$ ) is a continuous function for any $a \in A$. It will then follow that $\rho(A)$ is the cross-section algebra of a continuous field of $C^{*}$-algebras in the sense of $[3,10.1 .2]$. We will not prove this fact however, since we will not need it.

Now let $P$ be the set of all functions in $L^{\infty}(G, m)$ (complex valued) which have each $x \in G_{[\pi]}$ as period. Again this algebra is essentially a system of imprimitivity for the pair $\left(\tilde{\pi}, U^{\pi}\right)$, but we shall see that it is capable of discerning a finer structure than the algebra previously used. We write $\left[U_{x}\right]$ for $\left[U_{x}\right]_{x}$.

THEOREM 4.12. Let $G_{[\pi]}$ be closed. Then the commutant of $\left(\tilde{\pi}, U^{\pi}\right)$ intersected with the commutant of $P$ is the von Neumann algebra generated by the set of unitaries $\left[U_{x}\right], x \in G_{[\pi]}$, where $U_{x}$ is as defined in the proof of Lemma 4.11.

Proof. Proposition 4.8 shows that all $\left[U_{x}\right], x \in G$, are in the commutant of $\left(\tilde{\pi}, U^{\pi}\right)$. The periodicity of the functions in $P$ makes it clear that the $\left[U_{x}\right]$ are also in $P^{\prime}$. By Lemma 4.11, $\tilde{\pi}$ is unitarily equivalent with $\rho$ where $\rho(a)(g)=\pi\left(T\left(g_{c}\right) a\right)$ (notation as above). Now [28, 2, no. 3, Proposition 6 and no. 5, Lemma 4], shows that there is an essentially unique quasi-invariant measure $\lambda$ on $G / G_{[\pi]}$ and that a set in $G / G_{[\pi]}$ has measure zero if and only if its inverse image has Haar measure zero. It is then easy to see that if $\bar{\rho}(a): G / G_{[\pi]} \rightarrow H$ is defined by $\bar{\rho}(a)(\bar{g})=\rho(a)(g)$ $(\bar{g}=r(g)), \bar{\rho}$ is a representation of $A$ on $L^{2}\left(G / G_{[\pi]}, H, \lambda\right)$ by decomposable operators (see [4, Chapter 2]). Similarly $P$ induces a set of operators $\bar{P}$ on $L^{2}\left(G / G_{[\pi]}, H, \lambda\right)$. Clearly $\bar{P}$ is precisely the algebra of all scalar decomposable operators on $L^{2}\left(G / G_{[\pi]}, H, \lambda\right)$ (isomorphic with $L^{\infty}\left(G / G_{[\pi]}, \lambda\right)$ ). As indicated previously, any 
operator commuting with $\bar{P}$ is decomposable. If this operator also commutes with $\bar{\rho}$, then its value at each point $\bar{g}$ commutes with $\pi_{g}$ and is thus a scalar. It follows that the commutant of $\bar{\rho}$ and $\bar{P}$ on $L^{2}\left(G / G_{[\pi]}, H, \lambda\right)$ is just $\bar{P}$. Thus the von Neumann algebra generated by $\bar{\rho}$ and $\bar{P}$ is precisely the set of all decomposable operators.

Evidently this means that the von Neumann algebra generated by $\rho(A)$ and $P$ is the algebra $\mathscr{A}$ of all decomposables on $L^{2}(G, H, m)$ which have each $x \in G_{[\pi]}$ as a period.

Let $M$ be the set consisting of all scalar valued decomposable operators on $L^{2}(G, H, m)$ and all left translations $L_{x}, x \in G_{\pi}$. Then clearly $\mathscr{A}$ is the commutant of $M$ and so $M$ must generate $\mathscr{A}^{\prime}$. If $V$ is the unitary constructed in Lemma 4.11, then $V^{*} M V$ generates the commutant $\tilde{\pi}(A)^{\prime}$ of $\tilde{\pi}(A)$. We now determine $V^{*} M V$.

This isomorphism leaves the scalar decomposables invariant. On the other hand, if $L_{x}, x \in G_{[\pi]}$, is a translation, we have:

$$
\begin{aligned}
\left(V^{*} L_{x} V\right) f(g) & =\pi\left(\alpha\left(g_{s}, g_{c}\right)\right)^{*} U_{g_{s}}^{*} U_{x g_{s}} \pi\left(\alpha\left(x g_{s}, g_{c}\right)\right) f(x g) \\
& =\pi\left(\alpha\left(g_{s}, g_{c}\right)\right)^{*} c\left(g_{s}\right) U_{x} \pi\left(\alpha\left(x, g_{s}\right) \alpha\left(x g_{s}, g_{c}\right)\right) f(x g) \\
& =c\left(g_{s}\right) U_{x} \pi\left(\left(T(x) \alpha\left(g_{s}, g_{c}\right)^{*}\right) \alpha\left(x, g_{s}\right) \alpha\left(x g_{s}, g_{c}\right)\right) f(x g) \\
& =c\left(g_{s}\right) U_{x} \pi(\alpha(x, g)) f(x g) .
\end{aligned}
$$

The $c\left(g_{s}\right)$ exists for each $g$ because $U_{x}$ is unique only up to a constant of modulus one, and the corresponding function $c$ is measurable because of the measurability of the map $x \rightarrow U_{x}$ and the map $g \rightarrow g_{s}$. Thus $V^{*} L_{x} V=c\left[U_{x}\right]$, and so the commutant of $\tilde{\pi}$ is generated by the $\left[U_{x}\right], x \in G_{[\pi]}$, and the diagonalizables. Proposition 4.10 shows that all the $\left[U_{x}\right]$ also commute with all $U^{\pi}(g), g \in G$, and it is clear that the only diagonals which commute with all $U^{\pi}(g)$ are the constants. This completes the proof.

CoRollary 4.13. If $(T, \alpha)$ is a twisting pair for $(G, A)$, and $\pi$ is a representation of $A$ on $H$, then the system $\left(\tilde{\pi}, U^{\pi}, P\right)$ is irreducible (i.e. the commutant of all operators in $U^{\pi}(G), \tilde{\pi}(A)$, and $P$ consists of only multiples of the identity) if and only if $\pi$ is irreducible and $G_{[\pi]}=\{e\}$.

Proof. The necessity of the conditions follows from Proposition 4.8 (and Corollary 4.9), and the sufficiency follows from Theorem 4.12.

The most ideal situation would be to prove Theorem 4.12 and Corollary 4.13 without the presence of the algebra $P$. Mackey has conjectured that this result is incorrect even in the case of representations of group extensions, and recent results in the theory of direct integral representations of simple non type I $C^{*}$ algebras lead one to the same conclusion. Thus while we do not have a counterexample at this point, it seems likely that the above results are the best that one can prove in general. On the other hand, we can use a result of Blattner in certain cases to get the most desirable result.

Let $A$ be a $C^{*}$-algebra. Suppose that in addition to the standing hypotheses of 
this section, we also assume: If $x$ and $y$ are in $G$ and $\pi_{x}$ is not equivalent with $\pi_{y}$, then $\operatorname{ker}\left(\pi_{x}\right) \neq \operatorname{ker}\left(\pi_{y}\right)$. This holds in particular if $A$ is a type I algebra for then $\hat{A}=\operatorname{Prim}(A)$, but the condition does not require $A$ to be type $\mathrm{I}$. Then under these conditions, we have

THEOREM 4.14. The commutant of $\left(\tilde{\pi}, U^{\pi}\right)$ is generated by the set of unitaries $\left[U_{x}\right]$ as in Theorem 4.12.

We first prove a lemma, writing $B$ for the inner automorphisms by unitary double centralizers.

Lemma 4.15. Let $x_{0} \in G, a \in A$, and $\xi \in H$ (space of $\pi$ ) be such that

$$
\left\|\pi_{x_{0}}(a) \xi\right\|=\eta>0 .
$$

Then there exists a neighborhood $W$ of $x_{0}$ and a Borel mapping $u: W \rightarrow \mathscr{U}(H)$ such that if $x \in W,\left\|\pi_{x}(a) u(x)\right\|>\frac{1}{2} \eta$.

Proof. Let $\mathscr{A}=\operatorname{Aut}_{1}(A)$ and $D=\mathscr{A} / B$, with $p$ the natural projection. Then $\mathscr{A}$ in the strong topology is separable, metrizable, and complete (due to our standing hypotheses) and so is a polish space. Thus in particular any strongly open subset of $\mathscr{A}$ is a polish space. Also note that since $\mathscr{U}(M(A))$ is polish, [27, Lemma 3], guarantees the existence of a Borel cross-section $\alpha: B \rightarrow \mathscr{U}(M(A))$. Now let $a \in A$, $\xi \in H$ and consider the map $\beta: \mathscr{A} \rightarrow R^{+}$given by $\beta(z)=\|\pi(z(a)) \xi\|$. Then $\beta$ is strongly continuous. Since $\beta\left(T\left(x_{0}\right)\right)=\eta>0$, there is a neighborhood $U$ of $T\left(x_{0}\right)$ such that if $z \in U, \beta(z)>\frac{1}{2} \eta$. By remarks made above, $U$ is a polish space and the natural equivalence relation induced on $U$ by cosets of $B$ satisfies the hypotheses of [27, Lemma 2]. We conclude that there is a Borel cross-section $\psi: p(U) \rightarrow U$. Finally let $V=p^{-1} p(U)$ and $W=T^{-1}(V)$. As shown in 2.1.a, $p T$ is continuous, so $W=(p T)^{-1} p(U)$ is an open neighborhood of $x_{0}$. If $x \in W$ let $z_{x}=\psi p T(x)$, $\varphi(x)=T(x) z_{x}^{-1}$, and $r(x)=\alpha \varphi(x)$. Then $T(x) a=\varphi(x) z_{x}(a)=r(x) z_{x}(a) r(x)^{*}, z_{x}$ and $r(x)$ vary Borel measurably with $x$, and $z_{x} \in U$. If $u: W \rightarrow \mathscr{U}(H)$ is defined by $u(x)=\pi(r(x))$, then $\left\|\pi_{x}(a) u(x) \xi\right\|=\left\|u(x) \pi\left(z_{x}(a)\right) u(x)^{*} u(x) \xi\right\|=\left\|\pi\left(z_{x}(a)\right) \xi\right\|>\frac{1}{2} \eta$. This completes the lemma.

We now return to the proof of Theorem 4.14. The remainder of this proof is basically an adaptation of Blattner's proof of [29, Lemma 3]. We employ the notation of Theorem 4.12. It is enough to show that $P$ is in the weak closure of $\rho(A)$. For this it will suffice to prove that $\bar{P}$ is in the weak closure of $\bar{\rho}(A)$. Now $\bar{\rho}$ is a representation of $A$ which is the direct integral of irreducible inequivalent representations of $A$ on $H$ all with distinct kernels. $G$ acts as a topological transformation group on the $T_{0}$ space Prim $(A)$ (since it acts this way on $\hat{A}$ by Proposition 4.6) by sending $\operatorname{ker} \pi^{\prime}$ to $\operatorname{ker}\left(\pi^{\prime} \circ T\right)$ for each $\pi^{\prime}$ in $A$. There is thus induced in a natural way a one-to-one continuous mapping from $G / G_{[\pi]}$ to $\operatorname{Prim}(A)$ mapping $G / G_{[\pi]}$ onto the orbit of $I=\operatorname{ker}(\pi)$ in Prim $(A)$. Call this mapping $\varphi$, let $S$ be a closed subset of Prim $(A)$, and let $B=\varphi^{-1}(S)$. Let $Q$ be the projection on 
$L^{2}\left(G / G_{[\pi]}, H, \lambda\right)$ given by left multiplication by the characteristic function of $B$. We first prove that $Q$ is in the weak closure of $\rho(A)$. Let $f$ be a function in $L^{2}\left(G / G_{[\pi]}, H, \lambda\right)$ whose support is contained in $B$, and let $J$ be the ideal in $A$ given by $J=\bigcap S$. If $a \in J$, then $\pi_{x}(a) f(x)=0$ for all $x$ in $B$ and so $\tilde{\pi}(a) f=0$. Conversely suppose $f$ is continuous and support $f$ is not contained in $B$. Then $f$ is not zero in a neighborhood of some point $x$ which is not in $B$. Then $I \notin x^{-1}(S)$, and so $I \nsubseteq \bigcap x^{-1}(S)=K$. Since $\pi$ is irreducible, $\pi(K)$ is strongly dense in $B(H)$ and so we can find an $a^{\prime} \in K$ such that $\pi\left(a^{\prime}\right) f(x) \neq 0$. If $a=T(x)^{-1} a^{\prime}$ then $\pi_{x}(a) f(x)$ is not zero. Now Lemma 4.15 tells us that $\pi_{y}(a) f(x)$ is not zero in some neighborhood of $x$ and by the continuity of $f$ we must have $\pi_{y}(a) f(y)$ not zero in some neighborhood of $x$. Thus $\tilde{\pi}(a) f \neq 0$ and $a \in J$. We conclude that $Q$ is just the projection on the space of all $f$ in $L^{2}\left(G / G_{[\pi]}, H, \lambda\right)$ such that $\tilde{\pi}(a) f=0$ for all $a \in J$. This projection is well known to be in the weak closure of $\tilde{\pi}(A)$. One can easily prove that projections such as we considered above when we let $S$ be an arbitrary Borel set will form a projection valued measure, and so will be determined by knowing them on closed sets. One can therefore prove that if $S$ is any Borel set in Prim $(A)$, the characteristic function of $\varphi^{-1}(S)$ (acting as a projection) will be in the weak closure of $\tilde{\pi}(A)$. Finally a lemma of Blattner [29, Lemma 4] shows that the same conclusion holds for any Borel set in $G / G_{[\pi]}$. Hence all simple functions and so all of $P$ is in the weak closure of $\tilde{\pi}(A)$. It follows that $P$ is also in the weak closure of $\rho(A)$, and so we are done.

COROLlaRY 4.16. If the hypotheses of Theorem 4.14 hold, in particular if $A$ is a type I $C^{*}$-algebra, then if $\pi$ is any representation of $A$ on $H\left(\tilde{\pi}, U^{\pi}\right)$ is irreducible if and only if $\pi$ is irreducible and $G_{[\pi]}=\{e\}$.

We remark that Theorems 4.14 and 4.12 generalize theorems of Blattner (in the separable case) and Mackey respectively. However we get more information on the nature of the commutant of $\left(\tilde{\pi}, U^{\pi}\right)$ when this pair is not irreducible than is given in the aforementioned results, even in the group extension situation.

5. Representations from quasi-invariant traces. In this section we restrict our attention to twisted group algebras in which the cocycle $\alpha$ is trivial and the algebra $A$ is a von Neumann algebra. This is an important class of algebras, the representations of which, as we said before, have been studied under the name of covariant representations. We define quasi-invariant trace and show that each such trace defines in a natural way a representing pair for $(A, G)$.

Definition 5.1. Let $A$ be a von Neumann algebra on a Hilbert space $H$. A trace on $A$ is a function $\varphi: A^{+} \rightarrow[0,+\infty]$ such that:

(1) $\varphi(x+y)=\varphi(x)+\varphi(y) ; x, y \in A^{+}$,

(2) $\varphi(\lambda x)=\lambda \varphi(x) ; \lambda>0, x \in A^{+}$,

(3) $\varphi\left(u^{*} x u\right)=\varphi(x) ; x \in A^{+}, u$ unitary in $A$.

There are two-sided ideals $N_{\varphi}$ and $M_{\varphi}$ such that $M_{\varphi}^{+}=\operatorname{ker}_{\varphi}$ and

$$
N_{\varphi}^{+}=\left\{x \in A^{+} \mid \varphi(x)<\infty\right\} \text {. }
$$


We say that $\varphi$ is faithful if $M_{\varphi}=0$ and semifinite if $N_{\varphi}$ is weak operator dense in $A$. $N_{\varphi}^{1 / 2}$ is given an inner product by: $(x \mid y)=\dot{\varphi}\left(y^{*} x\right)$, where $\dot{\varphi}$ is the natural linear functional on $N_{\varphi}$ such that $\dot{\varphi} \mid N_{\varphi}^{+}=\varphi$. The completion of the prehilbert space $N_{\varphi}^{1 / 2} / M_{\varphi}^{1 / 2}$ is denoted by $L^{2}(\varphi)$. The natural representation $\pi_{\varphi}$ of $A$ on $L^{2}(\varphi)$ is the representation derived from left multiplication of $A$ on $N_{\varphi}^{1 / 2}$. If $\varphi$ is normal, the strong operator closures of both $N_{\varphi}$ and $M_{\varphi}$ have largest projections $E$ and $F$, $A$ is isomorphic with $A_{E} \oplus A_{F} \oplus A_{I-E}$, and $\varphi$ is infinite on $A_{I-E}$, zero on $A_{F}$ and faithful and semifinite on $A_{E}$. For all these facts see Dixmier [4].

Definition 5.2. Suppose $\varphi$ is a normal trace on a von Neumann algebra $A, G$ is a locally compact group, and $T: G \rightarrow$ Aut $(A)$ is a homomorphism. We say that $\varphi$ is $G$ quasi-invariant if both $N_{\varphi}$ and $M_{\varphi}$ are invariant under $T(G)$ (and so also $E_{\varphi}$ and $F_{\varphi}$ ).

Lemma 5.3. If $A$ is a von Neumann algebra, $\varphi$ is $G$ quasi-invariant on $A$ and $g \in G$, there are operators $x_{g}$ and $y_{g}$ in the center $C(A)$ of $A$ such that:

(1) $0 \leqq x_{g}, y_{g} \leqq 1$,

(2) $x_{g}^{2}+y_{g}^{2}=1$,

(3) $(T(g) \varphi)\left(x_{g}^{2} x\right)=\varphi\left(y_{g}^{2} x\right)$,

for all $x \in N_{\varphi}$ where $(T(g) \varphi)(x)=\varphi\left(T(g)^{-1} x\right)$.

Proof. By quasi-invariance and normality, we may assume that $\varphi$ is faithful and semifinite, and $N_{\varphi}$ is invariant.

Let $\chi=T(g) \varphi+\varphi$. Then $\chi$ is a semifinite normal trace on $A$ and $\varphi \leqq \chi$. So by $[4, \S 6,5.1]$, there is exactly one element $W_{g}$ in $C(A)$ such that $0 \leqq W_{g} \leqq 1$ and

$$
\varphi(x)=\chi\left(W_{g} x\right)=T(g) \varphi\left(W_{g} x\right)+\varphi\left(W_{g} x\right)
$$

for all $x \in N_{\varphi}$. Let $x_{g}$ and $y_{g}$ be the unique elements in $C(A)^{+}$such that $x_{g}^{2}=1-W_{g}$ and $y_{g}^{2}=W_{g}$. Then

$$
\varphi\left(W_{g} x\right)=\varphi\left(y_{g}^{2} x\right)=T(g) \varphi\left(\left(1-W_{g}\right) x\right)=T(g) \varphi\left(x_{g}^{2} x\right) .
$$

This completes the proof.

Now the center, $C(A)$, of $A$ is a commutative von Neumann algebra. By the Gelfand isomorphism we may identify $C(A)$ with the algebra of all continuous complex valued functions on a compact space $X$. Since $\varphi$ is faithful, it can be shown that $x_{g}$ and $y_{g}$ have support $I$, and so by the usual spectral arguments, we can find an increasing sequence $e_{1} \leqq e_{2} \leqq \cdots$ and numbers $\lambda_{i}>0,1 \leqq i<\infty$, such that $\sup _{i} e_{i}=1$ and $e_{i} x_{g} \geqq \lambda_{i} e_{i}$ for all $i$. There is then exactly one element $Z_{g}^{i} \in C(A)^{+}$for each $i$ such that $e_{i} Z_{g}^{i}=Z_{g}^{i}$ and $Z_{g}^{i} x_{g}=y_{g} e_{i}$.

LEMMA 5.4. Let $V_{n}^{g}$ be the sequence of operators in $L^{2}(\varphi)$ derived from the mappings $x \rightarrow T(g)^{-1}\left(Z_{g}^{n} x\right)$ of $\left(N_{\varphi}\right)^{1 / 2}$ into $\left(N_{\varphi}\right)^{1 / 2}$. Then the sequence $V_{n}$ is Cauchy in $L^{2}(\varphi)$ and converges to a unitary $U_{g}$ which is independent of the sequence $e_{1} \leqq e_{2} \leqq \cdots$ which determines the $Z_{g}^{n}$ 's. 
Proof. Let $e_{1} \leqq e_{2} \leqq \cdots$ and $f_{1} \leqq f_{2} \leqq \cdots$ be two increasing sequences of projections in $C(A)$ satisfying the properties given above. Let $\rho_{i j}=e_{i} f_{j}$ and let $Z_{g}^{i j}=Z_{g}^{i} f_{j}$. Then

$$
e_{i} f_{j} Z_{g}^{i j}=Z_{g}^{i j} \quad \text { and } \quad Z_{g}^{i j} x_{g}=y_{g} e_{i} f_{j} .
$$

On the other hand if $j$ is large enough, $Z_{g}^{i j}=Z_{g}^{i}$. Now let $x \in\left(N_{\varphi}\right)^{1 / 2}$. Then:

$$
\begin{aligned}
\| T(g)^{-1} & \left.\left(Z_{g}^{n} x\right)-T(g)^{-1}\left(Z_{g}^{i j} x\right) \|_{\varphi, 2}^{2} \quad \text { (where }\|\cdot\|_{\varphi, 2} \text { is the norm in } L^{2}(\varphi)\right) \\
& =\varphi\left(\left(T(g)^{-1}\left[\left(Z_{g}^{n}-Z_{g}^{i j}\right)\right)^{2}\left(x^{*} x\right)\right]\right) \\
& \left.=\varphi\left(T(g)^{-1}\left(\left(Z_{g}^{n}\right)^{2}-\left(Z_{g}^{i j}\right)^{2}\right)\left(x^{*} x\right)\right) \quad \text { (if } j \text { is sufficiently larger than } n\right) \\
& =(T(g) \varphi)\left(\left(\left(Z_{g}^{n}\right)^{2}-\left(Z_{g}^{i j}\right)^{2}\right)\left(x^{*} x\right)\right) \\
& =T(g) \varphi\left(x_{g}^{2}\left(1+\left(Z_{g}^{n}\right)^{2}\right) e_{n} x^{*} x-x_{g}^{2}\left(1+\left(Z_{g}^{i j}\right)^{2}\right) e_{i} f_{j} x^{*} x\right) \\
& =\varphi\left(y_{g}^{2}\left(1+\left(Z_{g}^{n}\right)^{2}\right) e_{n} x^{*} x-y_{g}^{2}\left(1+\left(Z_{g}^{i j}\right)^{2}\right) e_{i} f_{j} x^{*} x\right) \\
& =\varphi\left(e_{n} x^{*} x\right)-\varphi\left(e_{i} f_{j} x^{*} x\right) .
\end{aligned}
$$

If we replace $Z_{g}^{i j}$ by $Z_{g}^{m}$ in the above computation, we see that the sequence $V_{n}$ is Cauchy and so $U_{g}$ exists. On the other hand, the above shows that the same limit $U_{g}$ could also be defined by the $Z_{g}^{i j}$ arranged in a suitable sequence. Now $Z_{g}^{i j}=Z_{g}^{i} f_{j}$ $=Z_{g}^{j} e_{i}$ as is easily shown. By replacing the sequence $Z_{g}^{n}$ formed from the $e_{i}$ 's by the sequence formed from the $f_{j}$ 's, we can show that the limit $U_{g}$ is independent of the choice of projections. Finally a computation similar to the above shows that $\left\|U_{g} x\right\|_{\varphi, 2}^{2}=\varphi\left(x^{*} x\right)=\|x\|_{\varphi, 2}^{2}$ and since $U_{g}$ is invertible, it is unitary.

THEOREM 5.5. (a) $U_{g}^{*} \pi_{\varphi}(x) U_{g}=\pi_{\varphi}(T(g) x)$,

(b) $U_{g_{1}} U_{g_{2}}=U_{g_{1} g_{2}}$.

Proof. (a) If $y \in\left(N_{\varphi}\right)^{1 / 2}, x \in A$ then, writing $\pi$ for $\pi_{\varphi}$,

$$
\begin{aligned}
U_{g}^{*} \pi(x) U_{g} y & =U_{g}^{*}\left[\pi(x) \lim _{n}\left(T(g)^{-1}\left(Z_{g}^{n} y\right)\right)\right] \\
& =U_{g}^{*} \lim _{n} x T(g)^{-1}\left(Z_{g}^{n} y\right)=U_{g}^{*} \lim _{n} T(g)^{-1}\left(T(g)(x) Z_{g}^{n} y\right) \\
& =U_{g}^{*} U_{g}((T(g)(x) y)=(T(g) x) y=\pi(T(g) x) y .
\end{aligned}
$$

By continuity, the relation holds for all $y \in L^{2}(\varphi)$.

The defining relations show that $x_{g_{1} g_{2}}=x_{g_{1}} x_{g_{2}}$ and $y_{g_{1}} y_{g_{2}}=y_{g_{1} g_{2}}$.

Suppose that $\left(e_{i}\right), 1 \leqq i<\infty$, and $\left(f_{j}\right), 1 \leqq j \leqq \infty$, are sequences of projections in $C(A)$ used to define $U_{g_{1}}$ and $U_{g_{2}}$ respectively. Using $\left(e_{i} f_{j}\right), 1 \leqq i, j<\infty$, as the defining sequence for $U_{g_{1} g_{2}}$, we see that

and

$$
e_{i} f_{j} Z_{g_{1}}^{i} Z_{g_{2}}^{j}=Z_{g_{1}}^{i} Z_{g_{2}}^{j}
$$

Thus

$$
Z_{g_{1}}^{i} Z_{g_{2}}^{j} x_{g_{1} g_{2}}=Z_{g_{1}}^{i} Z_{g_{2}}^{j} x_{g_{1}} x_{g_{2}}=y_{g_{1}} y_{g_{2}} e_{i} f_{j}=y_{g_{1} g_{2}} e_{i} f_{j} .
$$

$$
U_{g_{1} g_{2}}(x)=\lim _{i j} T(g)^{-1}\left(Z_{g_{1}}^{i} Z_{g_{2}}^{j} x\right)=U_{g_{1}} U_{g_{2}}(x)
$$

for $x \in\left(N_{\varphi}\right)^{1 / 2}$ and by continuity we have (b). 
We now take up the consideration of conditions under which the representation given by the pair $\left(\pi_{\varphi}, U\right)$ constructed above is factor or irreducible. By quasiinvariance we have been able to assume that the trace $\varphi$ is faithful and semifinite, and so $L^{2}(\varphi)$ is simply the completion of the prehilbert space $N_{\varphi}^{1 / 2}$ with the inner product derived from $\varphi$.

Definition 5.6. If $B$ is a symmetric Banach algebra with identity and $G$ is a locally compact group acting on $B$ through a ${ }^{*}$-homomorphism $T$, then we say that $G$ is ergodic on $B$ if the only elements of $C(B)$ invariant under $T(g)$ for all $g \in G$ are multiples $\lambda I$ of the identity.

We say that $G$ is strongly ergodic on $B$ if the only two-sided ideals of $B$ invariant under $T(g)$ for all $g \in G$ are $\{0\}$ and $B$.

REMARK 1. Strong ergodicity clearly implies ergodicity, as we can see by forming the principle ideals generated by central elements.

REMARK 2. If $B$ is a von Neumann algebra, then each automorphism $T(g)$ of $B$ is ultraweakly continuous (see [4]) on $B$ and we can show that $G$ is strongly ergodic if and only if the only elements in $B$ invariant under all $T(g)$ are multiples of $I$. This is so because if $\mathscr{I} \subset B$ in an invariant ideal, the weak operator closure $\bar{I}$ of $\mathscr{I}$ in $B$ is also an invariant ideal. By [4, Chapter $1,3.3], \bar{I}$ is the principle ideal generated by its largest projection. This implies the result. In this case and in the case of ergodicity, for a von Neumann algebra, we may restrict our attention to invariant projections.

THEOREM 5.7. With notation as before, $\left(\pi_{\varphi}, U\right)$ is irreducible $\Leftrightarrow G$ is strongly ergodic on $A$.

Proof. Let $H$ be a subspace of $L^{2}(\varphi)$, invariant with respect to $\pi_{\varphi}(A)$. We first show that if we let

$$
H^{0}=\left\{x \in A \mid \pi_{\varphi}(x) H=\{0\}\right\}
$$

then $H^{0}$ is a closed, two-sided ideal in $A$ and

$$
H=\left\{\xi \in L^{2}(\varphi) \mid H^{0} \xi=\{0\}\right\} .
$$

The fact that $H^{0}$ is an ideal results immediately from the definitions and invariance of $H$ under $\pi_{\varphi}(A) . H^{0}$ is closed since $\pi_{\varphi}$ is continuous. Suppose $H^{0} \xi=\{0\}$ for some $\xi \in L^{2}(\varphi)$. Since $A$ is a ${ }^{*}$-algebra, $H^{\perp}$ (the subspace of $L^{2}(\varphi)$ perpendicular to $H)$ is invariant under $\pi_{\varphi}(A)$ and so also under $\pi_{\varphi}\left(H^{0}\right)$. Since $H^{0}$ is a $C^{*}$-algebra and $\pi_{\varphi}\left(H^{0}\right) \mid H^{\perp}$ is a nondegenerate representation, [3, 2.2.10], shows that $\pi_{\varphi}\left(a_{i}\right) \zeta \rightarrow \zeta$ for any $\zeta \in H^{\perp}$ where $\left(a_{i}\right)_{i \in I}$ is an approximate identity in $H^{0}$. Thus if $\zeta \in H^{\perp}$;

$$
(\xi, \zeta)=\lim _{i}\left(\xi, \pi_{\varphi}\left(a_{i}\right) \zeta\right)=\lim _{i}\left(\pi_{\varphi}\left(a_{i}^{*}\right) \xi, \zeta\right)=0 \quad \text { and } \quad \xi \in H^{\perp \perp}=H
$$

It is routine to show that $H$ is proper and nontrivial if and only if $H^{0}$ is proper and nontrivial. 
We claim that such an $H$ is invariant under $U_{g_{0}}$ if and only if $H^{0}$ is invariant under $T\left(g_{0}\right)$. In fact if $H$ is invariant under $U_{g_{0}}$, and $x \in H^{0}$, then $U_{g_{0}}^{*} \pi_{\varphi}(x) U_{g_{0}}(H)$ $=0$ and so $\pi_{\varphi}\left(T\left(g_{0}\right) x\right) H=0$ and $T\left(g_{0}\right) x \in H^{0}$. The converse is proved in the same way. Thus $L^{2}(\varphi)$ has no invariant subspaces under $U(G)$ and $\pi_{\varphi}(A) \Leftrightarrow$ there is no invariant ideal in $A$ except $\{0\}$ and $A$. This proves the theorem.

A theorem of Segal [20, Theorem 4.1], is a special case of the results of this section. The original construction of the representation $U$ from $\varphi$ was suggested by [16], and part of the proof of Theorem 5.7 was inspired by [20, Lemma 4.1.1].

Representations such as were constructed in the beginning of this section arise frequently. In particular, if $\varphi$ is a finite faithful trace on a von Neumann algebra $A$, it is quasi-invariant with respect to any group of automorphisms and so gives rise to a representing pair.

6. Computations. In this section we give the proofs of previous theorems which were left unproved.

THEOREM 2.2. Let $(T, \alpha)$ be a twisting pair for $(G, A)$ as in $\S 2$. Then $L^{1}(A, G ; T, \alpha)$ is a Banach *-algebra with multiplication and involution as given in $§ 2$.

Proof. (a) We show associativity:

$[(F \cdot K) \cdot H](g)$

$$
=\int_{G} \int_{G} F(\zeta)\left[\left[T(\zeta) K\left(\zeta^{-1} \xi\right)\right] \alpha\left(\zeta, \zeta^{-1} \xi\right)\right]\left[T(\xi) H\left(\xi^{-1} g\right)\right] \alpha\left(\xi, \xi^{-1} g\right) d \zeta d \xi
$$

On the other hand:

$$
\begin{aligned}
& {[F \cdot(K \cdot H)](g)} \\
& \quad=\int_{G} \int_{G} F(\zeta)\left\{\left[T(\zeta)\left[K(\xi)\left(T(\xi) H\left(\xi^{-1} \zeta^{-1} g\right)\right) \alpha\left(\xi, \xi^{-1} \zeta^{-1} g\right)\right]\right] \alpha\left(\zeta, \zeta^{-1} g\right)\right\} d \xi d \zeta \\
& \quad=\int_{G} \int_{G} F(\zeta)\left\{\left[T(\zeta)\left[K\left(\zeta^{-1} \xi\right)\left(T\left(\zeta^{-1} \xi\right) H\left(\xi^{-1} g\right)\right) \alpha\left(\zeta^{-1} \xi, \xi^{-1} g\right)\right]\right] \alpha\left(\zeta, \zeta^{-1} g\right)\right\} d \zeta d \xi
\end{aligned}
$$

Thus associativity reduces to showing

(1) $\left[T(\zeta)\left[K\left(\zeta^{-1} \xi\right)\left(T\left(\zeta^{-1} \xi\right) H\left(\xi^{-1} g\right)\right) \alpha\left(\zeta^{-1} \xi, \xi^{-1} g\right)\right]\right] \alpha\left(\zeta, \zeta^{-1} g\right)=$

(2) $\left[\left[T(\zeta) K\left(\zeta^{-1} \xi\right)\right] \alpha\left(\zeta, \zeta^{-1} \xi\right)\right]\left[\left(T(\xi) H\left(\xi^{-1} g\right)\right) \alpha\left(\xi, \xi^{-1} g\right)\right]$.

Now by the natural associativity between centralizers and the natural extension of $T$ to the double centralizer algebra, we get: for (1)

$$
\begin{aligned}
{\left[T(\zeta) K\left(\zeta^{-1} \xi\right)\right]\left[T(\zeta)\left[T\left(\zeta^{-1} \xi\right) H\left(\xi^{-1} g\right)\right]\right]\left[T(\zeta) \alpha\left(\zeta^{-1} \xi, \xi^{-1} g\right)\right] \alpha\left(\zeta, \zeta^{-1} g\right) } \\
=\left[T(\zeta) K\left(\zeta^{-1} \xi\right)\right]\left[T(\zeta)\left[T\left(\zeta^{-1} \xi\right) H\left(\xi^{-1} g\right)\right]\right] \alpha\left(\zeta, \zeta^{-1} \xi\right) \alpha\left(\xi, \xi^{-1} g\right) \\
=\left[T(\zeta) K\left(\zeta^{-1} \xi\right)\right] \alpha\left(\zeta, \zeta^{-1} \xi\right) T(\xi) H\left(\xi^{-1} g\right) \alpha\left(\xi, \xi^{-1} g\right)
\end{aligned}
$$

and by associativity this is (2). 
(b) We show $f^{* *}=f$ :

$$
\begin{aligned}
f^{* *}(x) & =\alpha\left(x, x^{-1}\right)^{*}\left[T(x) f^{*}\left(x^{-1}\right)^{*}\right] \Delta\left(x^{-1}\right) \\
& =\alpha\left(x, x^{-1}\right)^{*}\left\{T(x)\left[\alpha\left(x^{-1}, x\right)^{*}\left[T\left(x^{-1}\right) f(x)^{*}\right]\right]^{*}\right\} \Delta\left(x^{-1}\right) \Delta(x) \\
& =\alpha\left(x, x^{-1}\right)^{*}\left[T(x) T\left(x^{-1}\right)(f(x))\right]\left[T(x) \alpha\left(x^{-1}, x\right)\right] \\
& =\left[f(x) \alpha\left(x, x^{-1}\right)^{*}\right]\left[\alpha\left(x, x^{-1}\right) \alpha(e, x) \alpha(x, e)^{*}\right] \\
& =f(x) \alpha\left(x, x^{-1}\right)^{*} \alpha\left(x, x^{-1}\right)=f(x) .
\end{aligned}
$$

(c) We show $(f \cdot g)^{*}=g^{*} \cdot f^{*}$ :

$$
\begin{aligned}
\Delta(x)(f \cdot g)^{*}(x) & =\alpha\left(x, x^{-1}\right)^{*}\left[T(x) \int_{G} f(y)\left[T(y) g\left(y^{-1} x^{-1}\right)\right] \alpha\left(y, y^{-1} x^{-1}\right) d y\right] * \\
& =\int_{G} \alpha\left(x, x^{-1}\right)^{*}\left[T(x) \alpha\left(y, y^{-1} x^{-1}\right)^{*}\right]\left[T(x) T(y) g\left(y^{-1} x^{-1}\right)^{*}\right] \\
\times\left[T(x) f(y)^{*}\right] d y & \times\left[T(x) f\left(x^{-1} y\right)^{*}\right] d y \\
& =\int_{G} \alpha\left(x, x^{-1}\right)^{*}\left[T(x) \alpha\left(x^{-1} y, y^{-1}\right)\right]^{*}\left[T(x) T\left(x^{-1} y\right) g\left(y^{-1}\right)^{*}\right] \\
& =\int_{G} \alpha\left(x, x^{-1}\right)^{*}\left[\alpha\left(x, x^{-1} y\right) \alpha\left(y, y^{-1}\right) \alpha\left(x, x^{-1}\right)^{*}\right]^{*} \\
& \times \int_{G} \alpha\left(y, y^{-1}\right)^{*} \alpha\left(x, x^{-1} y\right)^{*}\left[T(x) T\left(y^{-1} y\right)^{*}\right]\left[T(x) f\left(x^{-1} y\right)^{*}\right] d y \\
& =\int_{G} \alpha\left(y, y^{-1}\right)^{*}\left[T(x) f\left(x^{-1} y\right)^{*}\right] d y
\end{aligned}
$$

On the other hand:

$$
\begin{aligned}
& \Delta(x)\left(g^{*} \cdot f^{*}\right)(x)= \int_{G} g^{*}(y)\left[T(y) f^{*}\left(y^{-1} x\right)\right] \alpha\left(y, y^{-1} x\right) d y \\
&= \int_{G} \alpha\left(y, y^{-1}\right)^{*}\left[T(y) g\left(y^{-1}\right)^{*}\right] \\
& \times\left\{T(y)\left[\alpha\left(y^{-1} x, x^{-1} y\right)^{*} T\left(y^{-1} x\right) f\left(x^{-1} y\right)^{*}\right]\right\} \alpha\left(y, y^{-1} x\right) d y \\
&= \int_{G} \alpha\left(y, y^{-1}\right)^{*}\left[T(y) g\left(y^{-1}\right)^{*}\right]\left[T(y) \alpha\left(y^{-1} x, x^{-1} y\right)^{*}\right] \\
& \times\left[T(y) T\left(y^{-1} x\right) f\left(x^{-1} y\right)^{*}\right] \alpha\left(y, y^{-1} x\right) d y \\
&= \int_{G} \alpha\left(y, y^{-1}\right)^{*}\left[T(y) g\left(y^{-1}\right)^{*}\right]\left[\alpha\left(y, y^{-1} x\right) \alpha\left(x, x^{-1} y\right)\right]^{*} \\
& \quad \times\left[\alpha\left(y, y^{-1} x\right)\right]\left[T(x) f\left(x^{-1} y\right)^{*}\right] d y \\
&=
\end{aligned}
$$


and so $(f \cdot g)^{*}=g^{*} \cdot f^{*}$. The proofs that $\|f \cdot g\| \leqq\|f\|\|g\|$ and $\left\|f^{*}\right\|=\|f\|$ are straightforward.

In Example 6, §2, the relevant computations were omitted. We review the setting. $0 \rightarrow H \stackrel{\sigma}{\rightarrow} G \stackrel{\pi}{\rightarrow} K \rightarrow 0$ is an exact sequence of locally compact groups. Identify $H$ with a subgroup of $G$ by means of $\sigma$.

Suppose $\pi$ has a Borel measurable right inverse (e.g. if $H$ is separable), and call it $\eta$. Let $d h, d g, d k$ be left Haar measures in $H, G$, and $K$ respectively.

Recall that:

For any $k_{1}, k_{2}$ in $K, \beta\left(k_{1}, k_{2}\right)=\eta\left(k_{1}\right) \eta\left(k_{2}\right) \eta\left(k_{1} k_{2}\right)^{-1}$.

$$
\text { For } k \text { in } K, \quad h \in H, \quad h^{k}=\eta(k)^{-1} h \eta(k) ; \quad h_{k}=\eta(k) h \eta(k)^{-1} \text {. }
$$

These operations are automorphisms of $K$, and there are positive real numbers $\delta(k)$ such that:

$$
d\left(h_{k}\right)=\delta(k) d h \quad \text { and } \quad d\left(h^{k}\right)=\frac{1}{\delta(k)} d h .
$$

(It should be carefully noted that neither the operations nor the function $\delta$ depend multiplicatively on $k$.)

(3) $\alpha\left(k_{1}, k_{2}\right) f(h)=f\left(\beta\left(k_{1}, k_{2}\right)^{-1} h\right) ;\left(f \alpha\left(k_{1}, k_{2}\right)\right)(h)=f\left(h \beta\left(k_{1}, k_{2}\right)^{-1}\right) \Delta\left(\beta\left(k_{1}, k_{2}\right)\right)^{-1}$ and finally

$$
(T(k) f)(h)=\delta(k)^{-1} f\left(h^{k}\right) .
$$

We prove that $(T, \alpha)$ is a twisting pair for $\left(K, L^{1}(H)\right)$.

Let $\Delta$ be the modular function of $H$ (i.e. $\left.d\left(h h_{0}\right)=\Delta\left(h_{0}\right) d h\right)$. Then: $\delta\left(k_{1} k_{2}\right)$ $=\delta\left(k_{1}\right) \delta\left(k_{2}\right) \Delta\left(\beta\left(k_{1}, k_{2}\right)\right)$. This is proved easily from the definitions and we will not show it. We also have, for any $x, y, z$ in $K$ :

$$
\beta(y, z)_{x} \beta(x, y z)=\beta(x, y) \beta(x y, z) .
$$

This is proved by using associativity of multiplication in $G$, as is well known, and again we leave the details to the reader.

We need to show that for any $x, y \in k$, that $\alpha(x, y)$ is a unitary double centralizer of $L^{1}(H), T(x)$ is an automorphism of $L^{1}(H)$, and the required relations between $T$ and $\alpha$ hold.

LEMMa 6.1. For any $k \in K, T(k)$ is a symmetric automorphism of $L^{1}(H)$.

Proof. The symmetry is obvious. Let $f, g \in L^{1}(H)$.

$$
\begin{aligned}
(T(k) f * T(k) g)(h) & =\int_{H}[T(k) f](\xi)[T(k) g]\left(\xi^{-1} h\right) d \xi \\
& =\int_{H} \delta(k)^{-2} f\left(\xi^{k}\right) g\left(\left(\xi^{-1} h\right)^{k}\right) d \xi=\int_{H} \delta(k)^{-2} f\left(\xi^{k}\right) g\left(\left(\xi^{k}\right)^{-1} h^{k}\right) d \xi \\
& =\int_{H} \delta(k)^{-1} f(\xi) g\left(\xi^{-1} h^{k}\right) d \xi=\delta(k)^{-1} f * g\left(h^{k}\right)=T(k)(f * g)(h) .
\end{aligned}
$$


The following lemma establishes the fact that $\alpha$ has values in the unitary double centralizers of $L^{1}(H)$.

LEMMA 6.2. If $h_{0} \in H$, let $\left(R_{h_{0}} f\right)(h)=\Delta\left(h_{0}^{-1}\right) f\left(h h_{0}^{-1}\right)$ and $\left(L_{h_{0}} f\right)(h)=f\left(h_{0}^{-1} h\right)$ define actions of $h_{0}$ on $L^{1}(H)$. Then $\Phi\left(h_{0}\right)=\left(L_{h_{0}}, R_{h_{0}}\right)$ defines a mapping from $H$ into the double centralizer algebra of $H$. Denote $L_{h_{0}} f$ by $h_{0} \cdot f$ and $R_{h_{0}} f$ by $f \cdot h_{0}$.

Proof. We must show that for any $f, g \in L^{1}(H),\left(f \cdot h_{0}\right) * g=f *\left(h_{0} \cdot g\right)$. Now for $h \in H$;

$$
\begin{aligned}
\left(f \cdot h_{0} * g\right)(h) & =\int_{H} f \cdot h_{0}(\xi) g\left(\xi^{-1} h\right) d \xi \\
& =\int_{H} f\left(\xi h_{0}^{-1}\right) \Delta\left(h_{0}^{-1}\right) g\left(\xi^{-1} h\right) d \xi \\
& =\int_{H} f(\xi) \Delta\left(h_{0}\right) \Delta\left(h_{0}^{-1}\right) g\left(h_{0}^{-1} \xi^{-1} h\right) d \xi \\
& =\int_{H} f(\xi) g\left(h_{0}^{-1} \xi^{-1} h\right) d \xi \\
& =\int_{H} f(\xi)\left(h_{0} \cdot g\right)\left(\xi^{-1} h\right) d \xi \\
& =f *\left(h_{0} \cdot g\right)(h) .
\end{aligned}
$$

The fact that $T$ and $\alpha$ satisfy the cocycle identity is simply a question of using the definitions and statement (5) above. We prove that for any $f \in L^{1}(H)$, $[T(x) T(y) f] \alpha(x, y)=\alpha(x, y)[T(x y) f]$. In fact:

$$
\begin{aligned}
{[T(x) T(y) f] \alpha(x, y)(h) } & =[T(x) T(y) f]\left(h \beta^{-1}(x, y)\right) \Delta\left(\beta(x, y)^{-1}\right) \\
& =f\left(\eta(y)^{-1} \eta(x)^{-1} h \beta^{-1}(x, y) \eta(x) \eta(y)\right) \Delta\left(\beta(x, y)^{-1}\right) \delta(x)^{-1} \delta(y)^{-1} \\
& =f\left(\eta(x y)^{-1} \beta^{-1}(x, y) h \eta(x y)\right) \delta(x y)^{-1} \\
& =\alpha(x, y)[T(x y) f](h) .
\end{aligned}
$$

Measurability considerations are clear and so $(T, \alpha)$ is a twisting pair.

We now show that $L^{1}(G)$ is isometrically isomorphic with $L^{1}\left(L^{1}(H), K ; T, \alpha\right)$.

LEMMA 6.3. Every element in $G$ can be uniquely written as $h \eta(k) ; h \in H, k \in K$. If $f \in L^{1}(G)$ then

$$
\int_{G} f(x) d x=\int_{K} \int_{H} \delta(k)^{-1} f(h \eta(k)) d h d k .
$$

Proof. If $\mathscr{K}(G)$ is the set of continuous complex valued functions with compact support on $G$, and $f \in \mathscr{K}(G)$, then $h \rightarrow f(h \eta(k))$ is continuous on $H$ and has compact support and so $\int_{H} f(h \eta(k)) d h$ exists. Now

$$
\begin{aligned}
\delta(k)^{-1} \int_{H} f(h \eta(k)) d h & =\delta(k)^{-1} \int_{H} f(\eta(k) h) d\left(h_{k}\right) \\
& =\int_{H} f(\eta(k) h) d h .
\end{aligned}
$$


Clearly this integral depends only on the coset of $\eta(k)$ by left invariance of $d h$, and it is also clear that

$$
F(k)=\int_{H} f(\eta(k) h) d h=\delta(k)^{-1} \int_{H} f(h \eta(k)) d h
$$

is a continuous function of $k$ with compact support whenever $f$ is such a function on $G$. By well-known arguments $\int_{G} f(x) d x=\int_{K} \int_{H} f(\eta(k) h) d h d k$ for all $f$ in $L^{1}(G)$, and this completes the lemma.

THEOREM 6.4. The mapping $\psi: L^{1}(G) \rightarrow L^{1}\left(L^{1}(H), K ; T, \alpha\right)$ given by:

$$
(\psi(f)(k))(h)=\delta(k)^{-1} f(h \eta(k))
$$

is an isometric isomorphism of Banach *-algebras.

Proof. The mapping is clearly linear. We show that it is isometric.

$$
\begin{aligned}
\|\psi(f)\| & =\int_{K}\|\psi(f)(k)\| d k \\
& =\int_{K} \int_{H}|(\psi(f)(k))(h)| d h d k \\
& =\int_{K} \int_{H} \delta(k)^{-1}|f(h \eta(k))| d h d k \\
& =\int_{G}|f(g)| d g=\|f\|, \quad \text { by Lemma 6.3. }
\end{aligned}
$$

We show that $\psi$ preserves multiplication. Let $f, g \in L^{1}(G)$. Then

$$
\psi(f) \cdot \psi(g)(k)=\int_{K}(\psi(f)(\rho))\left[T(\rho)\left(\psi(g)\left(\rho^{-1} k\right)\right)\right] \alpha\left(\rho, \rho^{-1} k\right) d \rho .
$$

Thus

$$
\begin{aligned}
&(\psi(f) \cdot \psi(g))(k)(h)=\int_{K} \int_{H} \psi(f)(\rho)(\xi)\left[\left(T(\rho) \psi(g)\left(\rho^{-1} k\right)\right) \alpha\left(\rho, \rho^{-1} k\right)\right]\left(\xi^{-1} h\right) d \xi d \rho \\
&=\int_{K} \int_{H} \psi(f)(\rho)(\xi) \Delta\left(\beta\left(\rho, \rho^{-1} k\right)\right)^{-1}\left[T(\rho) \psi(g)\left(\rho^{-1} k\right)\right] \\
& \cdot\left(\xi^{-1} h \beta\left(\rho, \rho^{-1} k\right)^{-1}\right) d \xi d \rho \\
&=\int_{K} \int_{H} \psi(f)(\rho)(\xi) \Delta\left(\beta\left(\rho, \rho^{-1} k\right)\right)^{-1} \delta(\rho)^{-1} \psi(g)\left(\rho^{-1} k\right) \\
& \cdot\left(\left(\xi^{-1} h\right)^{\rho}\left(\beta\left(\rho, \rho^{-1} k\right)^{\rho}\right)^{-1}\right) d \xi d \rho \int_{K} \int_{H} \delta(\rho)^{-1} f(\xi \eta(\rho)) \Delta\left(\beta\left(\rho, \rho^{-1} k\right)\right)^{-1} \delta(\rho)^{-1} \delta\left(\rho^{-1} k\right)^{-1} \\
& \cdot g\left(\left(\xi^{-1} h\right)^{\rho}\left(\beta\left(\rho, \rho^{-1} k\right)^{-1}\right)^{\rho} \eta\left(\rho^{-1} k\right)\right) d \xi d \rho
\end{aligned}
$$




$$
\begin{aligned}
& =\int_{K} \int_{H} \delta(\rho)^{-2} \delta\left(\rho^{-1} k\right)^{-1} \Delta\left(\beta\left(\rho, \rho^{-1} k\right)\right)^{-1} f(\xi \eta(\rho)) \\
& \cdot g\left(\eta(\rho)^{-1} \xi^{-1} h \eta(k)\right) d \xi d \rho \\
& =\int_{K} \int_{H} \delta(\rho)^{-1} \delta(k)^{-1} f(\xi \eta(\rho)) g\left((\xi \eta(\rho))^{-1} h \eta(k)\right) d \xi d \rho \\
& =\delta(k)^{-1} \int_{G} f(s) g\left(s^{-1} h \eta(k)\right) d s \\
& =\delta(k)^{-1} f * g(h \eta(k)) \\
& =\psi(f * g)(k)(h) .
\end{aligned}
$$

Let $\Delta_{H}, \Delta_{G}, \Delta_{K}$ be the modular functions in $H, G, K$ respectively (remembering that $d g$ was chosen relative to pregiven $d k$ and $d h$ so that $\psi$ would be isometric). We have previously referred to $\Delta_{H}$ as just $\Delta$. By using the definition of modular functions and relation (5) above we can show that for any $h \in H, k \in K$ we have:

$$
\Delta_{G}(h \varphi(k))=\Delta_{H}(h) \Delta_{K}(k) \delta(k)^{-1} .
$$

We omit the computation and proceed to the proof that $\psi$ preserves involution.

Let $f \in L^{1}(G), k \in K, h \in H$. Then

$$
\begin{aligned}
\psi(f)^{*}(k)(h) & =\Delta_{K}(k)^{-1} \alpha\left(k, k^{-1}\right)^{*}\left[T(k) \psi(f)\left(k^{-1}\right)^{*}\right](h) \\
& =\Delta_{K}(k)^{-1} \delta(k)^{-1} \psi(f)\left(k^{-1}\right)^{*}\left(\left(\beta\left(k, k^{-1}\right) h\right)^{k}\right) \\
& =\Delta_{K}(k)^{-1} \delta(k)^{-1} \Delta_{H}(h)^{-1} \Delta_{H}\left(\beta\left(k, k^{-1}\right)\right)^{-1} \overline{\psi(f)\left(k^{-1}\right)\left(\left(h^{-1} \beta\left(k, k^{-1}\right)^{-1}\right)^{k}\right)} \\
& \quad\left(\text { since for } x \in H, y \in K, \Delta_{H}\left(x^{y}\right)=\Delta_{H}(x)\right) \\
& =\Delta_{K}(k)^{-1} \Delta_{H}(h)^{-1} \delta\left(k^{-1}\right) \overline{\psi(f)\left(k^{-1}\right)\left(\eta(k)^{-1} k^{-1} \eta\left(k^{-1}\right)^{-1}\right)} \\
& =\Delta_{K}(k)^{-1} \Delta_{H}(h)^{-1} \overline{f\left((h \eta(k))^{-1}\right)} \\
& =\delta(k)^{-1} \Delta_{G}(h \eta(k))^{-1} \overline{f\left((h \eta(k))^{-1}\right)} \\
& =\delta\left(k^{-1}\right) f^{*}(h \eta(k))=\psi\left(f^{*}\right)(k)(h) .
\end{aligned}
$$

This completes the proof of Theorem 6.4.

We now prove Theorem 2.3. Let $(T, \alpha)$ be a twisting pair for $(A, G)$. Wendel has shown in [25] that if $m$ is an isometric double centralizer of $L^{1}(H)$ then there exists an element $h_{m} \in H$ and a complex number $\lambda_{m}$ of modulus one, both depending on $m$, such that for any $f \in L^{1}(H)$,

$$
(m f)(h)=\lambda_{m} f\left(h_{m}^{-1} h\right) \text { and }(f m)(h)=\lambda_{m} f\left(h h_{m}^{-1}\right) .
$$

Now for each $k_{1}, k_{2}$ in $K$, let $\beta\left(k_{1}, k_{2}\right)=h_{\alpha\left(k_{1}, k_{2}\right)}$ and let $\hat{\alpha}\left(k_{1}, k_{2}\right)=\lambda_{\alpha\left(k_{1}, k_{2}\right)}$. A simple computation shows that $\beta$ and $\hat{\alpha}$ both satisfy a cocycle relation with $T$ the mapping for $\beta$ and trivial automorphisms for $\hat{\alpha}$, and $(T, \beta)$ form a twisting pair for $\left(L^{1}(H), K\right)$. 
We can regard the mapping $\beta$ as a mapping into $H$ and therefore we may construct an abstract extension $G$ of $K$ by $H$ as in MacLane [19]. We now refer to Leptin [13] for the proofs of the statements to follow and we merely outline the rest of the argument. His cocycle is not precisely like ours, and he has abelian groups, but the proof he gives generalizes to our case.

If we provide $G$ with the product measure $m=d h \otimes d k$, where $d h$ and $d k$ are the Haar measures in $H$ and $K$ respectively (this is possible since $G=H \times K$ as a set), then we can show that $m$ is translation invariant and that the operations in $G$ are sufficiently measurable so that Weil's converse to Haar's theorem (see [24, A. 1]) applies. Then $G$ is dense in a unique locally compact group $\bar{G}$ and the restriction of Haar measure $d g$ in $\bar{G}$ to $G$ gives $m$. By examining the Weil topology, it can be seen that the topologies induced on $H$ and $K$ by being a subgroup and quotient group of $G$ respectively coincide with the original topologies. This quickly implies, since $G$ is complete, that $G=\bar{G}$ and the definitions of the operations show that $L^{1}(G)$ is isometrically isomorphic with $L^{1}\left(L^{1}(H), K ; T, \beta\right)$ in the manner indicsted in Example 6.

Now $\alpha=\hat{\alpha} \beta$, and if we have $f, g \in L^{1}\left(L^{1}(H), K ; T, \alpha\right)$, and

$$
\psi: L^{1}(G) \rightarrow L^{1}\left(L^{1}(H), K ; T, \beta\right)
$$

is the isomorphism given explicitly in Example 6, then for $f, g \in L^{1}(G)$ :

$$
\begin{aligned}
\psi(f) \cdot \psi(g)(k)(h) & =\int_{K} \int_{H} \psi(f)(\rho)(\zeta)\left[T(\rho) \psi(g)\left(\rho^{-1} k\right)\right]\left(\zeta^{-1} h\right) \beta\left(\rho, \rho^{-1} k\right) \hat{\alpha}\left(\rho, \rho^{-1} k\right) d \zeta d \rho \\
& =\int_{G} f(s) g\left(s^{-1} t\right) \hat{\alpha}\left(s, s^{-1} t\right) d s
\end{aligned}
$$

where $s=\zeta \eta(\rho): t=h \eta(k)$ and $\hat{\alpha}$ is considered as a cocycle on $G$, constant on cosets. This completes the proof.

We complete this section by showing that our induced representations correspond naturally to those of Mackey as stated in Theorem 4.2.

Proof of Theorem 4.2. As usual let

$$
0 \stackrel{\sigma}{\longrightarrow} H \stackrel{\pi}{\longrightarrow} \mathrm{H} \stackrel{\longrightarrow}{\longrightarrow} 0
$$

be an exact sequence of locally compact groups such that $\pi$ has Borel measurable left inverse $\eta$. Let $(T, \alpha)$ be as in Example $6, \S 1$. Let $u$ be a unitary representation of $H$ on a Hilbert space $\mathscr{H}$. Let $L^{2}(u)$ be the set of all measurable functions from $G$ to $\mathscr{H}$ such that:

(1) If $f \in L^{2}(u), x \in H, y \in G$, then $f(x y)=u(x) f(y)$.

(2) $\int_{K}\|f(x)\|^{2} d \dot{x}<\infty$ where $\dot{x}$ is the coset of $x$ and the integral makes sense because $\|f(x)\|$ is constant on cosets. The induced representation of $u$ in the sense of Mackey is the representation $U$ of $G$ on $L^{2}(u)$ given by (1):

$$
\left[U\left(g_{0}\right) f\right](g)=\Delta_{K}\left(\pi\left(g_{0}\right)\right)^{1 / 2} f\left(g g_{0}\right) .
$$


We note that $u$ induces a natural isomorphism $\Phi$ between $L^{2}(u)$ and $L^{2}(K, \mathscr{H}, d k)$ as follows:

If $F \in L^{2}(K, \mathscr{H}, d k), \Phi(F)(g) \equiv \Phi(F)(h \eta(k))=u(h) F(k)$.

The mapping $\Phi$ is easily seen to be measurable, bijective and linear. It is isometric by the defining conditions for $L^{2}(u)$. Thus $U$ corresponds in the usual way to a representation $\hat{U}=\Phi^{-1} U \Phi$ of $G$ on $L^{2}(K, \mathscr{H}, d k)$, and a standard computation using the definition of $\Phi$ shows that $\hat{U}$ is explicitly given by:

$$
\left[\hat{U}\left(g_{0}\right) F\right](k)=\Delta_{K}\left(k_{0}\right)^{1 / 2} U\left(\left(h_{0}\right)_{k} \beta\left(k, k_{0}\right)\right) F\left(k k_{0}\right)
$$

where $g_{0}=h_{0} \eta\left(k_{0}\right), \beta$ is considered to be $H$ valued, and all other notations are as in Example $6, \S 1$. We will freely use any facts from this example in what follows:

Let $\omega$ be the representation of $L^{1}(H)$ corresponding as usual to $u$, and let $\Pi$ be the representation of $L^{1}(G)$ corresponding to $\hat{U}$. As in Example 6, $\S 1$, we let $\psi$ be the usual isomorphism between $L^{1}(G)$ and $L^{1}\left(L^{1}(H), K ; T, \alpha\right) . \Pi \psi^{-1}$ is a representation of $L^{1}\left(L^{1}(H), K ; T, \alpha\right)$. We must show that $\Pi \psi^{-1}$ is the representation induced by $\omega$ in the sense of $\S 4$. If $H$ is embedded in the double centralizer algebra $M\left(L^{1}(H)\right)$ of $L^{1}(H)$ in the usual way, it will follow that the extension of $\omega$ to $M\left(L^{1}(H)\right)$, restricted to $H$ is just the original representation $u$, and so the pair $\left(\tilde{\omega}, U^{\omega}\right)$ induced from $\omega$ is explictly given by:

$$
[\tilde{\omega}(\theta) f](k)=\omega(T(k) \theta) f(k)=\int_{H}(T(k) \theta(h) u(h)) f(k) d h
$$

(where $\theta \in L^{1}(H)$ and $f \in L^{2}(K, \mathscr{H}, d k)$ )

and

$$
\left[U^{\omega}\left(k_{0}\right) f\right](k)=u\left(\alpha\left(k, k_{0}\right)\right) f\left(k k_{0}\right) \Delta_{K}\left(k_{0}\right)^{1 / 2} .
$$

Let $\Pi^{\prime}$ be the integrated form of $\left(\tilde{\omega}, U^{\omega}\right)$. We will show that $\Pi^{\prime} \psi=\Pi$ which will complete the proof. Let $f$ be an arbitrary element of $L^{1}(G)$ and $F$ an element of $L^{2}(K, \mathscr{H}, d k)$. Then:

$$
\begin{aligned}
{\left[\Pi^{\prime}(\psi(f)) F\right]\left(k_{0}\right) } & =\int_{K} \tilde{\omega}(\psi(f)(k))\left[U^{\omega}(k) F\right]\left(k_{0}\right) d k \\
& =\int_{K} \int_{H}\left\{T\left(k_{0}\right)[\psi(f)(k)]\right\}(h) u(h) u\left(\beta\left(k_{0}, k\right)\right) F\left(k_{0} k\right) \Delta_{K}(k)^{1 / 2} d h d k \\
& =\int_{K} \int_{H} \delta\left(k_{0}\right)^{-1} \Delta_{K}(k)^{1 / 2} \psi(f)(k)\left(\eta\left(k_{0}\right)^{-1} h \eta\left(k_{0}\right)\right) u\left(h \beta\left(k_{0}, k\right)\right) \\
& =\int_{K} \int_{H} \delta(k)^{-1} \delta\left(k_{0}\right)^{-1} \Delta_{K}(k)^{1 / 2} f\left(h^{k}{ }^{k} \eta(k)\right) u\left(h \beta\left(k_{0}, k\right)\right) F\left(k_{0} k\right) d h d k \\
& =\int_{K} \int_{H} \delta(k)^{-1} \Delta_{K}(k)^{1 / 2} f(h \eta(k)) u\left(h_{k_{0}} \beta\left(k_{0}, k\right)\right) F\left(k_{0} k\right) d h d k \\
& =\int_{G}[f(g) \hat{U}(g) F]\left(k_{0}\right) d g=[\pi(f) F]\left(k_{0}\right) .
\end{aligned}
$$




\section{REFERENCES}

1. R. F. Arens, The adjoint of a bilinear operation, Proc. Amer. Math. Soc. 2 (1951), 839-848. MR 13, 659.

2. L. Auslander and C. C. Moore, Unitary representations of solvable Lie groups, Mem. Amer. Math. Soc. No. 62 (1966). MR 34 \#7723.

3. J. Dixmier, Les $C^{*}$-algèbres et leurs représentations, Cahiers Scientifiques, fasc. 29, Gauthier-Villars, Paris, 1964. MR 30 \#1404.

4. — Les algèbres d'opérateurs dans l'espace hilbertien, Cahiers Scientifiques, fasc. 25, Gauthier-Villars, Paris, 1957. MR 20 \#1234.

5. S. Doplicher, D. Kastler and D. Robinson, Covariance algebras in field theory and statistical mechanics, Comm. Math. Phys. 3 (1966), 1-28. MR 34 \#4930.

6. E. Effros and F. Hahn, Locally compact transformation groups and $C^{*}$-algebras, Bull. Amer. Math. Soc. 73 (1967), 222-226. MR 38 \#1536.

7. J. Feldman and F. P. Greenleaf, Existence of Borel transversals in groups, Pacific J. Math. 25 (1968), 455-461. MR 37 \#6395.

8. J. Glimm, Families of induced representations, Pacific J. Math. 12 (1962), 885-911. MR 26 \#3819.

9. A. Grothendieck, Produits tensoriels topologiques et espaces nucléaires, Mem. Amer. Math. Soc. No. 16 (1955). MR 17, 763.

10. E. Hille and R. S. Phillips, Functional analysis and semi-groups, rev. ed., Amer. Math. Soc. Colloq. Publ., vol. 21, Amer. Math. Soc., Providence, R. I., 1957, MR 19, 664.

11. B. E. Johnson, An introduction to the theory of centralizers, Proc. London Math. Soc. (3) 14 (1964), 299-320. MR 28 \#2450.

12. G. P. Johnson, Spaces of functions with values in a Banach algebra, Trans. Amer. Math. Soc. 92 (1959), 411-429. MR 21 \#5910.

13. H. Leptin, Verallgemeinerte $L^{1}$-algebren, Math. Ann. 159 (1965), 51-76.

14. - Verallgemeinerte $L^{1}$-Algebren und projektive Darstellungen lokal kompakter Gruppen. I, Invent. Math. 3 (1967), 257-281. MR 37 \#5328.

15. - Verallgemeinerte $L^{1}$-Algebren und projektive Darstellungen lokal kompakter Gruppen. II, Invent. Math. 4 (1967), 68-86. MR 37 \#5328.

16. G. Mackey, Imprimitivity for representations of locally compact groups. I, Proc. Nat. Acad. Sci. U.S.A. 35 (1949), 537-545. MR 11, 158.

17. - Induced representations of locally compact groups. I, Ann. of Math. (2), 55 (1952), 101-139. MR 13, 434.

18. — Unitary representations of group extensions. I, Acta Math. 99 (1958), 265-311. MR 20 \#4789.

19. S. MacLane, Homology, Die Grundlehren der math. Wissenschaften, Band 14, Academic Press, New York and Springer-Verlag, Berlin and New York, 1963. MR 28 \#122.

20. I. E. Segal, A class of operator algebras which are determined by groups, Duke Math. J. 18 (1951), 221-265. MR 13, 534.

21. R. Schatten, A theory of cross spaces, Ann. of Math. Studies, No. 26, Princeton Univ. Press, Princeton, N. J., 1950. MR 12, 186.

22. M. Takesaki, Covariant representations of $C^{*}$-algebras and their locally compact automorphism groups, Acta Math. 119 (1967), 273-303. MR 37 \#774.

23. T. Turumaru, Crossed product of operator algebra, Tôhoku Math. J. (2) 10 (1958), 355-365. MR 21 \#1550.

24. A. Weil, L'intégration dans les groupes topologiques et ses applications, 2nd ed., Actualités Sci. Indust., no. 1145, Hermann, Paris, 1953. 
25. J. G. Wendel, Left centralizers and isomorphisms of group algebras, Pacific J. Math. 2 (1952), 251-261. MR 14, 246.

26. G. Zeller-Meier, Produits croisés d'une $C^{*}$-algèbre par un groupe d'automorphismes, C. R. Acad. Sci. Paris Sér. A-B 263 (1966), A20-A23. MR 33 \#7877.

27. J. Dixmier, Dual et quasi-dual d'une algèbre de Banach involutive, Trans. Amer. Math. Soc. 104 (1962), 278-283. MR 25 \#3384.

28. N. Bourbaki, Éléments de mathématiques, Livre VI: Intégration. Chapitre 7, Actualités Sci. Indust., no. 1306. Hermann, Paris, 1963. MR 31 \#3539.

29. R. Blattner, Group extension representations and the structure space, Pacific J. Math. 15 (1965), 1101-1113. MR 32 \#5785.

OAKLAND UNIVERSITY,

ROCHESTER, MichigAN 48063

Executive Office of the President,

Washington, D. C. 20504 